\title{
Observations of the Solar Corona from Space
}

\author{
Ester Antonucci $^{1}$ - Louise Harra ${ }^{2} \cdot$ Roberto Susino $^{1}$. \\ Daniele Telloni ${ }^{1}$
}

Received: 23 July 2020 / Accepted: 27 September 2020 / Published online: 19 October 2020

(C) The Author(s) 2020

\begin{abstract}
Space observations of the atmosphere of the Sun, obtained in half a century of dedicated space missions, provide a well established picture of the medium and large-scale solar corona, which is highly variable with the level of solar activity through a solar cycle and evolves with the long-term evolution of the magnetic cycles. In this review, we summarize the physical properties and dynamics of the medium and large-scale corona, consisting primarily of active regions, streamers and coronal holes; describe the dependence of coronal patterns on the magnetic field patterns changing through the solar cycle and the properties of the regions of open magnetic flux channeling the solar wind; the ubiquitous presence of fluctuations in the outer corona; the rotational properties of the large-scale corona; and the persistent hemispheric asymmetries in the emergence of magnetic fields and the distribution of the coronal emission.
\end{abstract}

Keywords Solar physics $\cdot$ Space observations $\cdot$ Solar corona $\cdot$ Solar wind

\section{Introduction}

The picture of the solar corona derived from ground-based and space observations is that of a hot inhomogeneous magnetized atmosphere, which is constantly evolving and consisting of features developing on different scales in the various phases of the solar activity cycle. Active regions are dominant in the ascending phase of the solar cycle and at solar maximum when the relatively simple poloidal configuration of the solar magnetic field typically observed at solar minimum is disrupted by the emergence of the toroidal field of the new cycle. Coronal holes and quiescent streamers, reflecting long-lived large-scale patterns, are

E. Antonucci

ester.antonucci@inaf.it

1 Istituto Nazionale di Astrofisica (INAF), Osservatorio Astrofisico di Torino, Via Osservatorio, 20, 10125, Pino Torinese (TO), Italy

2 Physikalisch-Meteorologische Observatorium Davos/World Radiation Center and Eidgenössische Technische Hochschule-ETH Zürich, Zürich, Switzerland 
prevalent in the declining to minimum phase of solar activity when the magnetic field becomes organized according to lower order multipoles. When the level of activity increases, magnetic energy is sporadically released in sudden and powerful reconnection events occurring at coronal heights. These events are characterized by the emission of highly energetic electromagnetic radiation and particle acceleration, frequently associated with huge plasma ejections, inducing transient changes and a reorganization of the structure of the corona.

The X-ray image of the Sun, obtained by the Naval Research Laboratory scientists during a sub-orbital rocket flight on April 19, 1960, in the form of a crude low-resolution photograph, revealed for the first time the hottest layers of the solar atmosphere against the bright disk. The solar corona appeared as a highly inhomogeneous X-ray source with $80 \%$ of the emission coming from 5\% or less of the area of the solar disk (Blake et al. 1963; Friedman 1986). Friedman reports the adventurous account of the first pioneering observations of the solar X-ray sources. By the early seventies, after a decade of monitoring the solar emission in the ultraviolet and $\mathrm{X}$ electromagnetic domains, the main characteristics of the structure and dynamics of the corona were fairly well established thanks to the Skylab mission, preceded by observations with the Orbiting Solar Observatories and by a series of sub-orbital rocket flights carrying scientific instrumentation. X-rays turned out to be principally emitted from coronal loops, regions of closed magnetic field lines overlying zones of enhanced photospheric magnetic field, which were recognized as the basic elements of the solar corona (Vaiana et al. 1973; Rosner et al. 1978a). The faintest coronal features, with a plasma density one or two orders of magnitude lower than in active regions and characterized by a predominantly open magnetic field, were named coronal holes. Concomitantly, it was recognized that magnetic fields are not only acting to confine the plasma in the inner part of the solar atmosphere but they are also playing a fundamental role in coronal heating. Rosner et al. (1978b) pointed out that two elements are crucial to produce a hot corona, namely, a relatively strong magnetic field coupled with an input of mechanical energy into the field. At approximately the same time on the basis of the OSO VIII observations, Athay and White (1978) and Athay and White (1979) established that the acoustic waves originating in the photosphere are stopped before reaching the corona, thus supporting the view of a coronal heating process strictly related to the presence of magnetic fields. At the rate the solar atmosphere is heated up to a million-degree temperature, global gravitational confinement was recognized to be insufficient to prevent coronal plasma to undergo a steady expansion (Parker 1958), thus giving origin to an incessant wind streaming outwards in the interplanetary space where it has been detected with in situ instrumentation since the very beginning of the space era. At last, spectroscopic observations obtained with the Solar and Heliospheric Observatory, SOHO (Domingo et al. 1995), directly showed that in the outer layers of the solar atmosphere, above a few solar radii from Sun center, wind outflows fill the entire corona.

In the eighties, the full electromagnetic spectrum of the active corona, spanning from the ultraviolet emission to gamma rays, was explored at high spectral resolution with the spectrometers of the Solar Maximum Mission, SMM, launched in 1980. The main scientific results of the mission are summarized in the volume 'The Many Faces of the Sun' (Strong et al. 1999). A few of these instruments were also imaging active regions in the UV and soft X-ray range and - for the first time - in the hard X-ray domain in order to identify the sites where energy is suddenly released during flares. A series of successful space missions, SMM, P78 (Doschek 1983) and Hinotori (Tanaka 1981), operating almost simultaneously during the maximum of solar cycle 21 , allowed an in-depth study of the flaring activity of the Sun. The existence of extremely high plasma temperatures, that can reach values of the order of $30-40 \mathrm{MK}$ in the coronal region where energy is released, was discovered thanks to the 
first observations of spectra emitted by the Fe XXVI hydrogen-like ion (Parmar et al. 1981; Tanaka et al. 1982). These missions clearly revealed the crucial role played by magnetic reconnection in the sudden and powerful release of energy during solar flares.

The first global images of the solar corona, spanning from the inner layers observed close to the limb in the Fe XIV emission line to the outermost regions observed in white light, were obtained in 1996 with the suite of the three visible light coronagraphs forming the SOHO Large Angle Spectroscopic Coronagraph (LASCO; Brueckner et al. 1995) that extended the coverage of coronal imaging out to $30 R_{\odot}$. LASCO observations, obtained from the Lagrangian point L1, ensured an almost uninterrupted monitoring of the corona for more than two solar cycles. Moreover, the high sensitivity of the LASCO instruments allowed the detection of quite faint coronal mass ejections, opening a new window on the understanding of the large-scale coronal reconfiguration during and in response to coronal activity. At the same time, the Ultraviolet Coronagraph Spectrometer (UVCS; Kohl et al. 1995) on SOHO, obtained the first direct observations of the continuous expansion of the corona by measuring the outflow velocity of the solar wind (Kohl et al. 1997) at least out to $5 R_{\odot}$ (Telloni et al. 2007a). The UVCS results on coronal outflows were achieved by detecting the Doppler dimming of resonantly scattered ultraviolet emission lines, such as the $\mathrm{H}$ I Lyman- $\alpha$ at $121.6 \mathrm{~nm}$ line and the $\mathrm{O}$ VI 103.2, $103.7 \mathrm{~nm}$ doublet.

In the last two solar cycles, namely 23 and 24, almost uninterrupted observations of the full disk photospheric magnetic field, first with the Michelson Doppler Imager (MDI; Scherrer et al. 1995) on SOHO and later on with the Helioseismic and Magnetic Imager (HMI; Scherrer et al. 2012) of the Solar Dynamics Observatory (SDO; Pesnell et al. 2012), allowed us to infer with unprecedented accuracy the evolution of the coronal magnetic field which is shaping the solar atmosphere. In turn, the corona was monitored with a comprehensive set of instruments allowing for an excellent coverage from its inner layers, observed on disk in the ultraviolet domain, to its outermost region detected at the limb in ultraviolet and visible light. The inner corona was monitored with a series of instruments: the Extreme-Ultraviolet Imaging Telescope (EIT; Delaboudiniere et al. 1995) of the SOHO mission, the Transition Region And Coronal Explorer telescope (TRACE; Strong et al. 1994; Handy et al. 1999), the Atmospheric Imaging Assembly (AIA; Lemen et al. 2012) on board SDO, the Extreme Ultraviolet Imager (EUVI-SECCHI; Howard et al. 2008) on board STEREO (Kaiser et al. 2008), and the Sun Watcher with Active Pixels and Image Processing (SWAP) on board Proba-2 (Seaton et al. 2013). At the limb, the outer corona was observed out to $30 R_{\odot}$, namely the edge of the SOHO coronagraphs field-of-view, and from different perspectives by combining SOHO and STEREO observations. In the same years, coronal composition, density, temperature and velocity fields were measured on disk with the spectrometers of several missions: Yohkoh (Culhane et al. 1991), SOHO (Harrison et al. 1995; Wilhelm et al. 1995), Hinode (Culhane et al. 2007). This unprecedented powerful combination of space instruments allowed us to study the solar atmosphere at its different scales through more than two solar cycles, that, interestingly enough, were separated by the anomalously long and weak solar minimum of cycle 23 , thus allowing us to highlight the striking differences characterizing the last two solar cycles.

The next sections focus, from the observational point of view, on the principal coronal features and properties with emphasis on the large-scale solar corona:

- active regions, the medium-scale coronal features which are the principal manifestation of the emergence of the toroidal component of the solar magnetic field;

- the large-scale coronal structures, primarily coronal holes and quiescent streamers, manifestation of the poloidal component of the field whose influence is progressively increasing in the declining phase of the cycle and during solar minimum; 
- solar wind in the coronal acceleration region;

- asymmetries related to the coronal/heliospheric current sheet; and

- coronal rotation, phenomena strictly related to the magnetic field patterns emerging from the photosphere and shaping the solar atmosphere.

The flaring and eruption activity related to huge sporadic mass and energy releases occurring in the corona are not discussed in this paper.

\section{Active Regions: Structure, Heating, and Input into the Solar Wind}

Magnetic fields are well accepted as being key in heating the Sun's atmosphere, generating the solar wind, and releasing energy rapidly in the form of solar flares. Active regions are an important magnetic feature on the Sun that are usually associated with sunspots, which are locations of strong magnetic fields. In this section we will discuss the structure of active regions, how the heating is sustained, and the evidence for the contribution of plasma from active regions into the solar wind. There is a wealth of information on this topic, and this brief summary only touches the surface. A living review article on modeling and observations of coronal loops by Reale (2014) and coronal heating by De Moortel and Browning (2015) are available for further details. Input from the corona into the slow solar wind is reviewed by Abbo et al. (2016).

\subsection{Active Region Structure and Heating}

The structure of an active region is defined by the magnetic field. Due to the sparsity of coronal magnetic field measurements, the photospheric magnetic field is commonly used to constrain and model the coronal magnetic field. This has challenges due to the widely different plasma regimes that exist between the photosphere that is characterized by a high plasma $\beta$ and into the corona that has a low plasma $\beta$. The plasma dominates in the photosphere, whereas the magnetic field does so in the corona. There are many ways of addressing the difficult task of determining the coronal field from photospheric measurements. These have been summarized by Wiegelmann et al. (2017) in a review paper, and they review magnetohydrodynamic models, magnetohydrostatic, force-free and potential field models. An example of non-linear force free field (NLFFF) model is shown in Fig. 1. Warren et al. (2018) have carried out a systematic study of 15 magnetically different active regions. In total they studied 12202 loops that were traced in the coronal images from SDO AIA. The purpose was to determine which model most accurately described the structure of the loops, and by how much the observations differed from the models. They found that the differences in the extrapolations were small, with the nonlinear force free field model being preferable. The difference in angle between the observed loops and the modeled loops was found to be between $5^{\circ}-12^{\circ}$.

It is clear that the active region loops highlight the magnetic field in the corona. But what do the loops themselves consist of, and at what spatial resolution will isothermal structures be revealed? As coronal instrumentation has improved over the years, more evidence is leading to the suggestion that the loops at coronal temperatures are close to being resolved. This is critical in order to understand the process(es) of heating the loops. In the small-scale reconnection scenario for example, simulations predict current sheets forming on the size scales of 100s meters (Shay et al. 2003). Whereas evidence from both spectroscopic and imaging data is suggesting that the loops are 100s kilometers in width. The filling factors of 

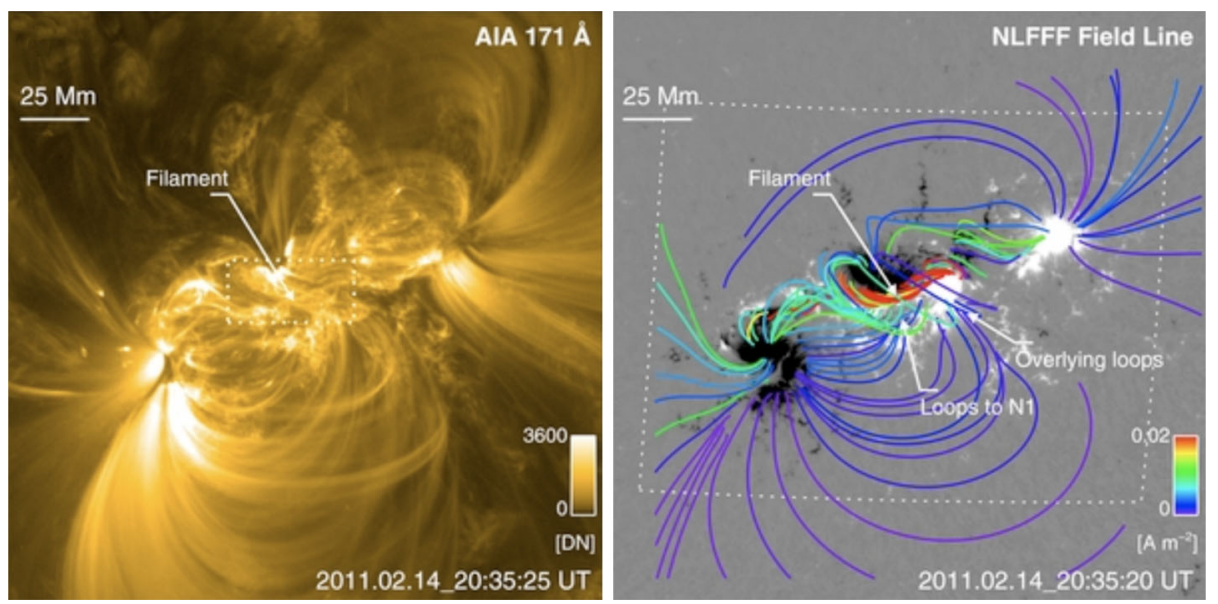

Fig. 1 The left image is from AIA 171-band showing coronal magnetic structures. The right image shows the NLFFF extrapolation plotted over a cutout from the vertical magnetic field map. The lines are color-coded by the vertical current density at their footpoints (see the color bar); red field lines correspond to strong current density. In this active region there was enough free energy for an X-class flare to occur. Figure from Sun et al. (2012)

loops can be determined spectroscopically giving an indication of the structure within, and whether there are multi-threads within an observed loop. Evidence for a filamentary nature of the corona was suggested by Martens et al. (1985) and Linford and Wolfson (1988), who inferred a width of the filaments of the order of 100-300 km by comparing the density of the coronal plasma derived on the basis of density-sensitive line ratios with the results obtained by combining the plasma emission measure and the scale of the soft X-ray emission region, by using SMM observations. Del Zanna and Mason (2003) use the high resolution TRACE data along with SOHO Coronal Diagnostic Spectrometer (CDS) data, and argue that the smallest threads seen by TRACE do not have many structures within them suggesting that the loop size is close to the spatial resolution of TRACE at $\approx 365 \mathrm{~km}$. Warren et al. (2018) using SDO/AIA data found isolated active region loops that have narrow temperature distributions - but are still not isothermal. The filling factor in this case was $10 \%$ which is consistent with the multi-thread models and also spatial resolutions of hundreds $\mathrm{km}$. The highest spatial resolution achieved for the corona was with the rocket flight High Resolution Coronal Imager $(\mathrm{HiC})$ with a resolution of $\approx 150 \mathrm{~km}$. Brooks et al. (2013) analyzed 91 loops and measured the Gaussian widths to be $\approx 270 \mathrm{~km}$. Figure 2 shows an example of coronal loops as seen by SDO/AIA and HiC. The temperature distributions of these loops were found to be very narrow. This is consistent with the active region coronal structure being at a scale that is well above the spatial scale of proposed physical heating mechanisms. Modeling was carried out by Aschwanden and Peter (2017) to discriminate between resolved and unresolved loops. They found that the loops are fully resolved in $\mathrm{HiC}$ data, which agrees with Brooks et al. (2013). It also is in agreement with photospheric granulation sizes supporting heating mechanisms on the macroscopic scale.

There are two physical mechanisms that are thought to contribute to the structure and heating of active regions. These are based on the magnetic fields in the active regions, one is based on magnetic reconnection of stressed field lines, and the other on the dissipation of energy from magnetohydrodynamic waves. There are theories, simulations and observations that support and describe both mechanisms. 
Fig. 2 The top left figure shows an AIA image, and the top right shows a HiC image. The intensity profiles are shown below. The loop seen by AIA is composed of two loops when observed by HiC. Adapted from Brooks et al. (2013)
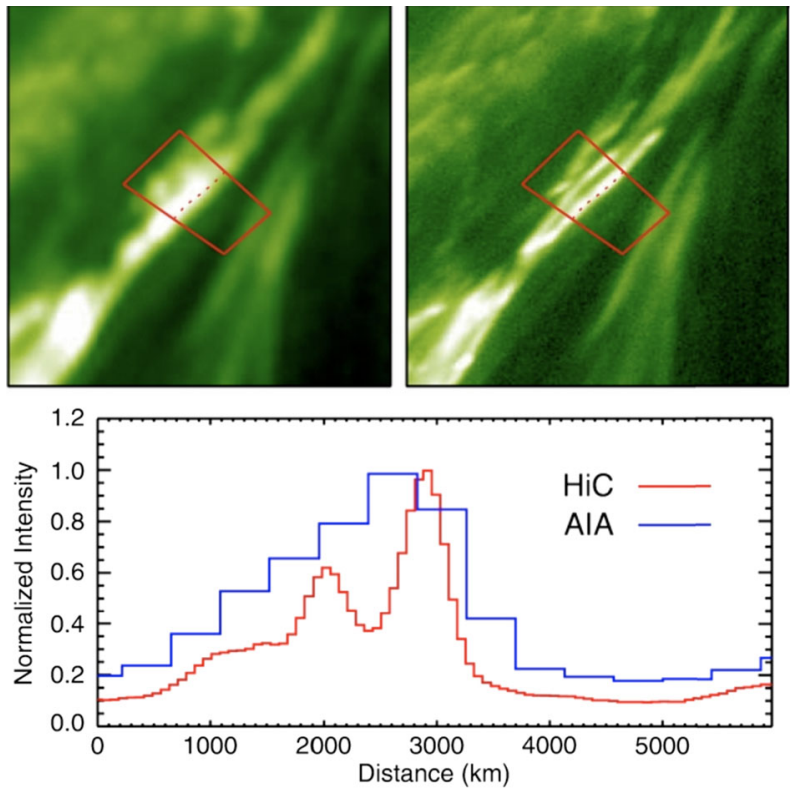

Magnetic Reconnection This is predicted to be caused through the braiding and tangling of magnetic field lines within a loop (Parker 1972). Observing such braiding is very challenging, with evidence being seen with the HiC observations (Cirtain et al. 2013, see Fig. 2). Simulations have been developed by e.g. Pontin et al. (2017) that explore what the signatures would be in the observations due to energy release caused by braiding. They show that braided magnetic field lines do not necessarily lead to the appearance of braiding in the intensities observed in the corona. The appearance of braiding observationally is dependent on the extent of the braiding. Another key measurement is evidence of hard X-ray emission which would be expected if the plasma is being heated impulsively. This is a challenging observation for such small flares, as opposed to their large energy release counterparts of solar flares. Recent instrumentation such as the Focusing Optics X-ray Solar Imager (FOXSI) rocket (Glesener et al. 2016) made such observations of an active region. FOXSI observed a quiescent active region and X-ray spectra were modeled for nanoflare sequences with different characteristics such as heating amplitudes and durations (Marsh et al. 2018). The FOXSI data were consistent with nanoflare sequences. This confirms that impulsive heating is taking place in these coronal loops.

Dissipation of Magnetic Waves Magnetohydrodynamic waves may be produced by the interaction of the magnetic field with convective flows clearly observed in the photosphere, as first suggested by Alfvén (1947). These waves can propagate into the atmosphere and dissipate their energy, heating the corona. Waves are observed ubiquitously in the corona. Tomczyk et al. (2007) detected upward propagating waves using the Coronal Multi-Channel Polarimeter (CoMP) instrument. CoMP allows measurements of intensity, line-of-sight velocity and an estimate of direction of the magnetic field. The waves propagate in the direction of the magnetic field. Van Doorsselaere et al. (2008) demonstrates that the CoMP observational findings can be explained in terms of kink magneto-acoustic waves. Although waves are seen clearly in the corona, the estimate of the energy they carry and available for coronal 

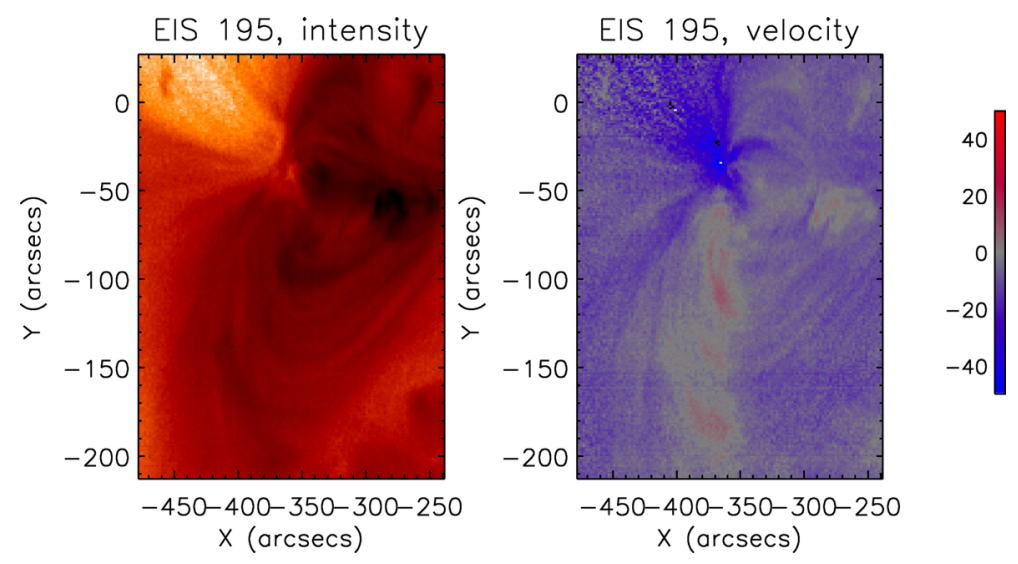

Fig. 3 The left image shows the intensity of the Fe XII emission line. The right image shows the Doppler velocity. The strongest blue-shifts are in the regions of lower intensity at the edges of the active region (Harra et al. 2008)

heating is a difficult task. De Pontieu et al. (2007) studied the movement of jet-like features in the chromosphere and estimated energies, comparing with MHD simulations. They found that these Alfvén waves observed in the chromosphere could possibly heat the corona. Models have been developed to explain the observations. An example by van Ballegooijen et al. (2017) explored Alfvén wave turbulence and whether it can heat the corona. They found that for waves launched from the photosphere, there can be enough heat to maintain a temperature of $2.5 \mathrm{MK}$, which is lower than temperatures measured in active regions cores $(\approx 3-4 \mathrm{MK})$. Their model also predicts observables such as line widths, which are higher than observed values. Improvements in the model are being explored. The accuracy of predictable observables in modeling continues to progress, and these developments are being explored alongside developments in the observations. It is clear there is evidence for both waves and reconnection. To make further steps new observations that will come online in the 2020s will help constrain and open up new areas in our understanding.

\subsection{Active Region Input to the Slow Solar Wind}

The origin of the slow solar wind remains to be fully understood. There are various options, and in this section we will look at the evidence of slow solar wind sources from active regions. Yohkoh observations have been used to establish a mass loss contributing to the solar wind coming from the outward expansion of coronal active regions (Uchida et al. 1992), thus suggesting active regions as possible sporadic sources of solar wind. Observations from the Hinode-EUV Imaging Spectrometer (EIS) have consistently shown evidence of blue-shifted plasma at the edges of active regions (Harra et al. 2008). Figure 3 shows an example of the blue-shifted material. Different explanations have been given for the physical explanation of the upflows, including waves, reconnection in the corona (Baker et al. 2009) and reconnection from below (De Pontieu et al. 2007). The plasma is clearly moving outwards, but the question is whether this plasma can make it all the way into the solar wind. This is a non-trivial problem to confirm. One way to do so is through modeling, an example of which was carried out by Edwards et al. (2016). In this work seven active regions were studied, and the upflows and composition were measured. The structure of the magnetic field was determined from modeling to identify sites of open magnetic field where this plasma could 

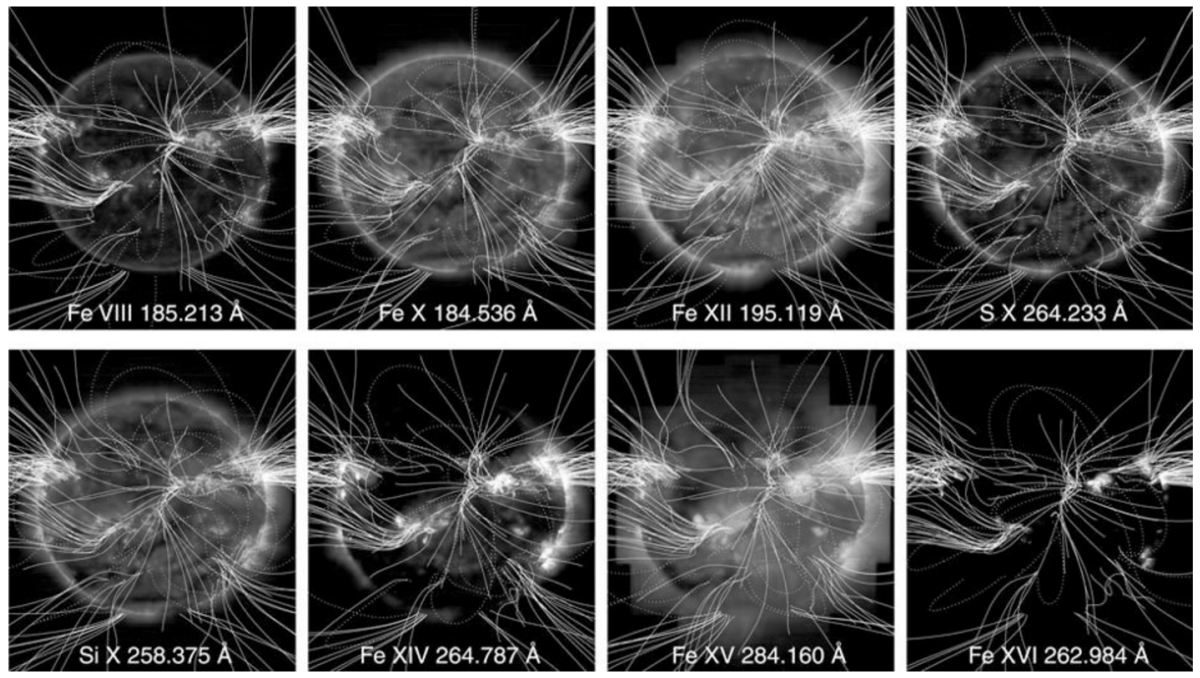

Fig. 4 Magnetic fields lines from a potential field source surface (PFSS) calculation are overlaid on the full-Sun intensity images from Hinode-EIS. The solid lines are open and the dotted lines are closed. Each pixel in these images has a spectrum which allows the Doppler velocity and abundance to be determined, and the regions corresponding to sources of solar wind to be determined (Brooks et al. 2015)

freely flow into the solar wind. In most cases these upflows did not in fact correspond to locations of open field, so it cannot be assumed that if an upflow is observed this will lead into the solar wind. However, the sites of upflow were intersected by separatrix surfaces with null points located high in the corona. This indicates that these regions could be sites of reconnection that would have an impact on a large scale.

Another way to determine possible sources into the solar wind is to explore the composition, and compare that with in-situ measurements. Imaging spectrometers have a small fieldof-view - around the size of an active region normally. Solar wind can emanate from multiple regions, so a large field of view is beneficial for understanding the solar wind sources. One way to explore this is to run full Sun rasters, which are time consuming but provide spectroscopic information across the whole Sun. This takes a couple of days and is now carried out by both Hinode-EIS instrument and Interface Region Imaging Spectrometer (IRIS) on a regular basis. Brooks et al. (2015) analyzed such a dataset. They determined the Doppler velocity and the composition measurements and combined these with global magnetic field models. This allowed the sources of the solar wind to be determined, some of which are active regions as can be seen in Fig. 4. This is a powerful tool, although observationally and analytically time-consuming.

\subsection{Future Observations of Active Regions}

The 2020s will see new datasets coming from the Daniel K. Inouye Solar Telescope, DKIST, and the Solar Orbiter space mission. DKIST is a $4 \mathrm{~m}$ telescope and will observe the Sun with the highest ever spatial resolutions. In addition, it will have accurate measurements of flows and magnetic fields - through the photosphere, chromosphere and potentially into the corona. On the same timescales the Solar Orbiter mission will be observing at its closest point to the Sun at $0.28 \mathrm{AU}$. This viewpoint will allow high spatial resolution imaging 
and spectroscopy to understand active regions in more depth and at the same time be close enough to the Sun to measure the solar wind at a location in its evolution that we can determine the linkage back to the Sun. Hand in hand with this, theory and models are making huge progress, becoming not only a way to understand physical processes but being interactively involved with observation planning on missions.

\section{Large-Scale Corona: Coronal Structures, Coronal Wind, Hemispheric Asymmetries, Coronal Rotation}

Solar eclipses offered the only way to observe the large-scale corona out to many solar radii until space coronagraphs were made available. The first extensive space observations of the large-scale corona were obtained with the white light coronagraph (MacQueen et al. 1974) of the Skylab mission, imaging the Sun's atmosphere at the limb from 1.5 to $6 R_{\odot}$. The outer corona was continually monitored for 9 months in the time frame 1973-1974, this allowing an unprecedented study of its morphology and evolution over several solar rotations. The first coronal transient - a phenomenon nowadays labeled as 'coronal mass ejection' - was detected with sufficient space and time coverage on June 10, 1973. Thanks to the Skylab suite of instruments, the event, activated by an eruptive prominence, could be described in its rapid transit from the lower corona, observed at ultraviolet and X-ray wavelengths, to the outer corona out to $6 R_{\odot}$, in the white light. At the time, the dimension of the eruption, bigger than the disk of the Sun, as well as its rapid outward expansion were quite surprising and unexpected. The coronal images at the limb, combined with the X-ray pictures of the inner corona seen against the solar disk, greatly contributed to understand the large-scale pattern of the solar atmosphere primarily composed by structures such as streamers and coronal holes, regions characterized by closed and open magnetic fields, respectively. Furthermore, these observations highlighted the physical connection between the solar atmosphere and the heliosphere, in particular, between coronal holes and the high-speed solar wind streams observed in the interplanetary space.

\subsection{Large-Scale Coronal Structures}

\subsubsection{Closed Magnetic Field Regions: Coronal Streamers}

In its inner part, the inhomogeneous solar atmosphere is shaped by the bright active regions (see Sect. 2), whilst the emission of the outer corona is modulated by large streamers, extending out to a few solar radii. The physical properties, geometry and solar cycle dependence of coronal streamers, as mainly derived from solar eclipse observations, have been reviewed by Koutchmy and Livshits (1992) just before the beginning of the SOHO era, when the observation of the global corona obtained with an unprecedented spatial coverage from 1.1 out to $30 R_{\odot}$ became possible thanks to the LASCO suite of coronagraphs and when the vantage point of these coronagraphs, the Lagrangian point L1, ensured an almost uninterrupted monitoring of the solar corona for more than two solar cycles.

The first LASCO images obtained in 1996 are an excellent example of the typical solar minimum configuration of the atmosphere of the Sun, attained when active regions are rare. The undisturbed corona is shaped by the large-scale magnetic field and its emission is dominated by equatorial streamers, forming a belt around the Sun. Polar coronal holes are present at high latitudes. The inner corona, observed with LASCO C1 in the Fe XIV emission at $530.3 \mathrm{~nm}$, consisted of a complex of sub-streamers formed by two persistent bright 


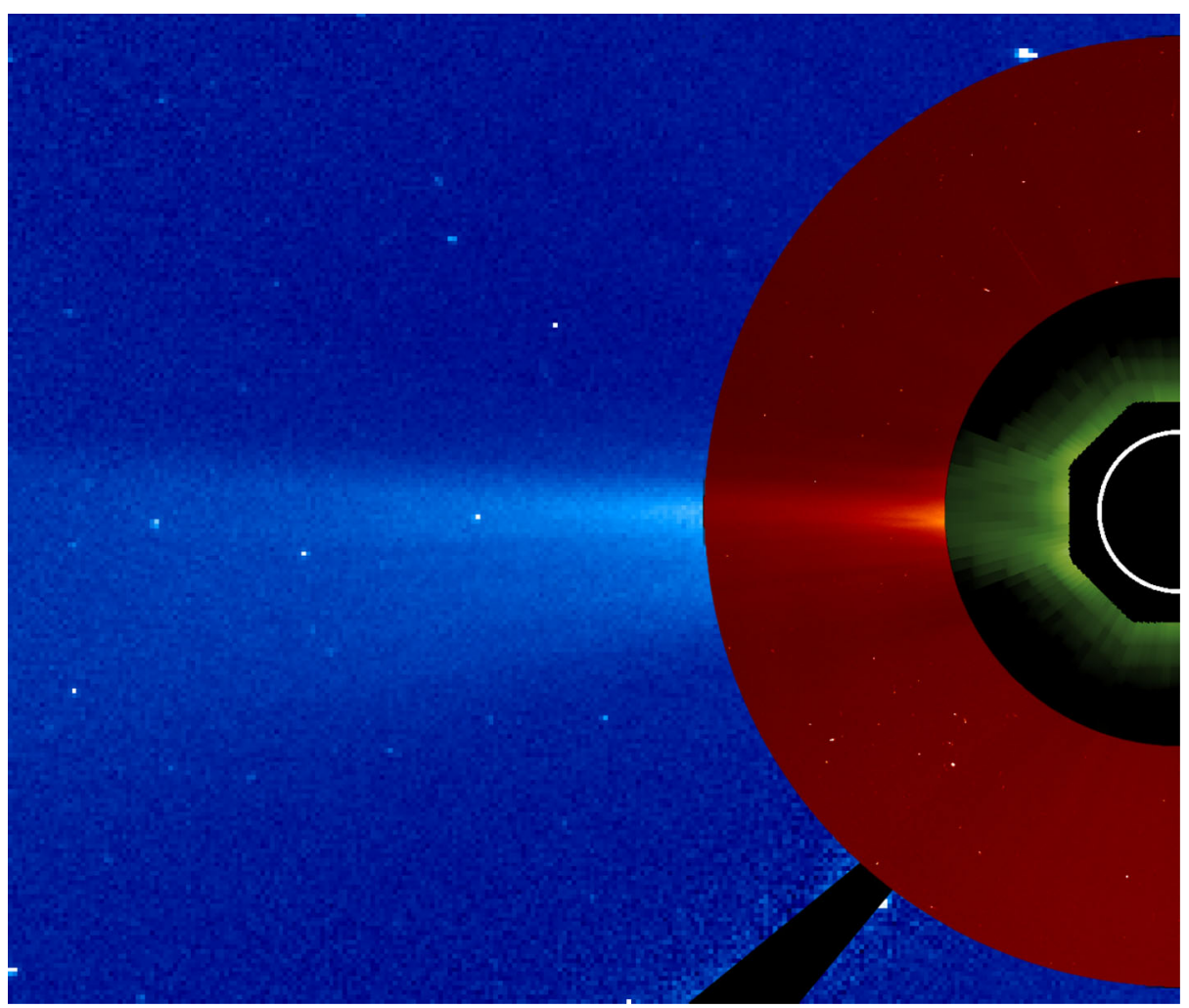

Fig. 5 A composite image of the O VI 103.2 nm emission measured with UVCS on August 10, 1996, at the east limb between 1.5 and $3 R_{\odot}$ (green), the $\mathrm{C} 2$ white-light image obtained on the same day between 3 and $6 R_{\odot}$ (red), and the $\mathrm{C} 3$ white-light image above $6 R_{\odot}$ (blue). The three images have been scaled to the spatial resolution of the $\mathrm{C} 2$ image (11.4 $\mathrm{arcsec} / \mathrm{pixel})$

closed loop systems at mid latitudes, $30^{\circ}-45^{\circ}$, and a more diffuse and variable equatorial structure. Beyond $2 R_{\odot}$, the mid latitude features converged toward the equator to form a large equatorial streamer stretching out to $5 R_{\odot}$, continuing as a single high-density sheet near the equator, observed in white light (Schwenn et al. 1997; Schwenn 2000). In this phase of the solar cycle, no Fe XIV emission was detected at the polar caps, sites of large polar coronal holes, due to their low temperature $\leq 1 \mathrm{MK}$ (Wilhelm et al. 1998).

Figure 5 shows the typical solar minimum corona observed on August 10, 1996, at the end of cycle 22, with the LASCO C2 and C3 coronagraphs and with the UVCS coronagraph spectrometer in the $\mathrm{O}$ VI $103.2 \mathrm{~nm}$ emission below $3 R_{\odot}$. The axis-symmetric magnetic dipole becomes dominant in the outer corona, beyond $3 R_{\odot}$, due to the rapid fall-off with height of higher order multipoles of the solar magnetic field prevalent in the inner corona and responsible for the more complex configuration at mid and low latitudes. During 1996, structural changes of the large-scale corona were observed to occur fairly gradually over a prolonged period of time, except for a transient and variable equatorial extension of the northern polar hole (Del Zanna and Bromage 1999). The dipolar configuration characteristic of the solar minimum corona shown in Fig. 5 can be found in some of the eclipse drawings collection by Loucif and Koutchmy (1989), where the extended equatorial streamer belt was well developed in the years 1954 and 1976, that is, in the last phase of solar cycles 18 and 20. 
The equatorial thin and dense layer, seen along the streamer axis in the LASCO C3 field of view (Schwenn et al. 1997), outlines the formation of the heliospheric current sheet, embedded in a density layer, that separates the open magnetic field lines of opposite polarities emerging from the polar coronal holes and stretched outward by the solar wind flow (see also Fig. 5). At solar minimum, this layer in general lies approximately in the solar equatorial plane, as first suggested on the basis of the interplanetary magnetic field observations (Rosenberg and Coleman 1969; Schulz 1973). During the successive minimum phase of cycle 23 , the polar dipole moment was significantly weaker and the magnitude of the polar field, 0.6 Gauss, was lower than that measured in the previous minimum, 1.0 Gauss (Kirk et al. 2009). Probably for this reason, the solar corona never quite reached a persistent simple dipolar configuration characterized by a streamer belt confined near the equatorial plane and the heliospheric current sheet started to flatten only in mid-2008, 2009 (Wang et al. 2009).

High-speed ( $\geq 700 \mathrm{~km} \mathrm{~s}^{-1}$ ) wind streams emerging from the core of polar coronal holes were encountered by Ulysses in its out of ecliptic journey at all latitudes except in the range $\pm 21^{\circ}$ in heliographic latitude, where the heliospheric current sheet was embedded in the slow wind flowing at $\sim 400 \mathrm{~km} \mathrm{~s}^{-1}$. In the interplanetary regions magnetically connected to the core of the polar coronal holes, where the fast wind flows, only a slight gradual increase of the wind speed, from 5 to $10 \%$, was observed with increasing heliolatitude. At that time, the heliosphere, reflecting the configuration of the corona, was rather symmetric and the heliospheric current sheet was tilted by about $7^{\circ}$ to $8^{\circ}$ with respect to the heliographic equator. This heliospheric configuration was basically sustained for a period of time as long as half a solar cycle (Woch et al. 1997).

The streamer belt observed in the first year of SOHO scientific operations has been object of extensive observation campaigns, analysis and modelling efforts (see Li et al. 1998; Gibson et al. 1999a,b, and references therein). Streamer structures were found to be close to hydrostatic equilibrium and fairly isothermal from $1.1 R_{\odot}$ to $1.5 R_{\odot}$, with temperatures of about $1.5-1.6 \times 10^{6} \mathrm{~K}$ and densities around $1.3 \times 10^{7} \mathrm{~cm}^{-3}$, in the center of a streamer, and the same physical conditions can persist for several days ( $\mathrm{Li}$ et al. 1998; Gibson et al. 1999a). Results obtained by studying streamers in the rising and maximum phases of cycle 23 (Parenti et al. 2000; Uzzo et al. 2004, 2007), compared with those obtained at solar minimum of cycle 22 , show higher densities, by 2-3 times, and higher temperatures by about $10 \%$ in the streamer inner part (Uzzo et al. 2007).

Coronal Streamers During the Solar Cycle At solar maximum, the mid latitude coronal streamers do not converge any longer to generate a large equatorial belt associated with an almost equatorial high-density current sheet, but form separate thin current sheets rising radially from their source regions. Their thickness can be as small as $0.14 \times 10^{6} \mathrm{~km}$ at $4 R_{\odot}$. Compact and unstable streamers are sometime associated with active regions (Morgan and Habbal 2010).

Streamers frequently enclose structures not observable in coronal lines due to their relatively low temperature. These features, seen as prominences above the solar limb and as filaments on the disk, are complex regions of cool and dense material immersed in the coronal plasma which is two orders of magnitude hotter and more tenuous. They are located above polarity inversion lines where the radial component of the photospheric magnetic field changes sign and are held against gravity at coronal heights by the surrounding magnetic field. The characteristics of prominences have been reviewed by many authors, e.g., by Labrosse et al. (2010), Mackay et al. (2010), Parenti (2014), and Vial and Engvold (2015). Prominences and filaments can be fairly stable regions. These quiescent structures retain photospheric composition according to Parenti et al. (2019). Quiescent filaments, that may 
exist at all latitudes, at solar minimum are preferentially located at high latitudes, $45^{\circ}-50^{\circ}$, and often form a crown around the polar caps. When solar activity increases, coronal streamers move towards the poles according, for instance, to the observations near solar maximum by Zhukov et al. (2008). Near the poles, extended fan-shaped structures observed in the EUV, persisting for several rotations, are either associated with the open field lines overlying prominences (Seaton et al. 2013) or related to pseudostreamers that separate holes of the same polarity (Wang et al. 2007).

According to a large-scale hemispheric pattern of the magnetic field, filaments contain dominant negative helicity - dextral filaments - in the northern hemisphere and positive helicity - sinistral filaments - in the southern hemisphere. This pattern remains substantially the same when the solar polar magnetic field reverses, that is, the helicity hemispherical distribution is unchanged from cycle to cycle (Rust 1967; Martin et al. 1994; Yeates et al. 2007; Mackay et al. 2010). The eruptive phase of prominences associated with coronal mass ejections - the key mechanism to avoid unbounded accumulation of magnetic helicity in the solar corona (Low 2003, and references therein) -, a fundamental chapter of the physics of the active Sun, is not discussed here.

As the large-scale corona evolves in response to the evolution of the magnetic field of the Sun, changing from a quasi-dipolar configuration at sunspot minimum to a more complex topology at sunspot maximum, the coronal and heliospheric current sheets warp and assume a more complex geometry (Schulz 1973). As a consequence, multiple current and density sheets may appear in the corona-heliosphere global system (Morgan and Habbal 2010; Wang 2014). For instance, from extrapolations of the photospheric magnetic field Wang et al. (2014) have inferred that during 2012, close to the maximum of cycle 24, the heliospheric current sheet was split into two distinct structures with circular cross sections, centered near the heliographic equator and roughly $180^{\circ}$ apart in longitude. This configuration was ascribed to the dominance of the Sun's non axis-symmetric quadrupolar component during the magnetic reversal of cycle 24 .

A study of the global long-term coronal conditions over most of the activity cycle 24 , from solar minimum to maximum, performed on the basis of the EUV emission of the solar corona detected by SDO-AIA, shows that during the years 2011-2017 the coronal mean temperature rises from 1.4 to $1.8 \mathrm{MK}$, whereas the emission measure increases by almost a factor of $50 \%$. An increased high-temperature component near $3 \mathrm{MK}$ related to activity drives the increase in the coronal mean temperature at solar maximum, whilst the bulk of the plasma remains approximately at the same temperature. In the same period, the mean quiet Sun magnetic field increases from $1.6 \mathrm{G}$ in 2011 to $2.0 \mathrm{G}$ in 2015 (Morgan and Taroyan 2017).

Pseudo-Streamers and Associated Heliospheric Density Sheet Generally, the magnetic structure of coronal streamers consists of a single or an odd number of bright coronal loop systems that the solar wind stretches to form a quasi-radial sheet dividing field lines of opposite polarity. In recent years, observers highlighted the importance of pseudo-streamers, coronal structures connected to an even number of bipoles that separate field lines of the same polarity (Wang et al. 2007). Normally, they are high-latitude structures forming between a polar coronal hole and a small mid-latitude one in the same solar hemisphere. The specific configuration of pseudo-streamers was clearly depicted by Schatten et al. (1969) when showing an example of computation of the coronal magnetic field derived from the source surface model on the basis of Mount Wilson Observatory observations of the photospheric field. Unlike the case of classical streamers giving origin to heliospheric current sheets, the outward extensions of pseudo-streamers are simply heliospheric plasma sheets, 
since they divide field lines of the same polarity. They are observed at the limb just as thin bright rays, being the closed loop system at their base usually more confined near the solar surface than in streamers.

Comparing the physical properties of a pseudo streamer and of a bipolar streamer, both observed in March 2008 with UVCS, Abbo et al. (2015) found that beyond $1.95 R_{\odot}$ the pseudo-streamer under study exhibits lower density and a tendency toward higher outflow velocity of the $\mathrm{O}$ VI ions than the simple bipolar streamer, as predicted by Wang et al. (2007). This occurs at a height where an increase in ion kinetic temperature of the oxygen ions, likely related to a higher level of energy deposition, is also observed. In this study, the velocity of the solar wind is directly measured in the corona by applying the Doppler dimming technique.

A further characteristics of pseudo-streamers is that they do not emit plasma blobs the propagating coronal inhomogeneities identified by Sheeley et al. (1997) - as streamers do, due to their peculiar magnetic configuration. According to Sheeley et al. (2009) coronal blobs are flux ropes presumably formed by reconnection of the gradually expanding streamer loops with each other. The convergence of open field lines of the same polarity above the pseudo-streamer impedes the outward expansion of the underlying coronal loops, as suggested by Wang et al. (2012).

\subsubsection{Open Magnetic Field Regions: Coronal Holes}

During several rocket experiments carried out in the sixties, the observations against the solar disk in extreme ultraviolet and X-rays emitted by the coronal plasma revealed regions that appear as 'holes' due to their very low emission, with characteristics in antithesis of active regions. The appearance of regions characterized by strongly dimmed coronal lines was recognized to be primarily due to the drop estimated to be by a factor of 3 in the coronal electron pressure and a coronal temperature lower than $6 \times 10^{5} \mathrm{~K}$ with respect to the quiet Sun conditions (Munro and Withbroe 1972). A spectacular coronal hole extending longitudinally from the northern to the southern polar cap was photographed in X-rays on November 24, 1970 during a sounding rocket flight. The divergence of the soft X-ray structures surrounding the hole suggested that this large region of reduced emission and temperature was the site of open magnetic fields. This interpretation was confirmed by the results of an extrapolation to coronal heights of the photospheric field that in the hole was observed to be weak and predominantly unipolar. The extrapolated coronal field turned out to be radial in the center of the hole and arching away from the hole at the boundaries (Krieger et al. 1973). Furthermore, this region was identified by Krieger et al. (1973) as the source of a recurrent high velocity solar wind stream observed in the interplanetary space. Noci (1973) showed that the energy escaping into space from coronal holes and converted into energy of the solar wind was indeed sufficient to power a 'very strong' solar wind.

The Skylab observations, in the period from May 1973 through January 1974, raised further interest in these newly discovered coronal features. A large persistent coronal hole with longitudinal extension from the northern polar cap to the southern hemisphere, known as CH1 - the 'boot of Italy' - was detected over several solar rotations (Timothy et al. 1975). Its repetitive passage on disk could be related to the recurrent fast wind streams detected in the heliosphere and giving origin to geo-magnetic disturbances. The correlation of the coronal hole area and magnetic field polarity with the speed and polarity of the hole-associated wind stream, respectively, further confirmed that large coronal holes are open magnetic structures, sources of the high velocity solar wind streams (Nolte et al. 1976). Furthermore, the discovery of the chain of phenomena from the coronal source to the associated wind stream and 
its geo-effects solved the mystery of the recurrent geomagnetic disturbances, until then attributed to the so called 'M regions', not yet univocally identified on the Sun (Hundhausen 1972; Neupert and Pizzo 1974). A systematic study of the properties of coronal holes observed in the XUV lines during the Skylab mission was carried out by Bohlin (1977) and Bohlin and Sheeley (1978).

Withbroe (1988) presented an exhaustive summary of the coronal hole physical conditions, such as coronal densities and temperatures, kinetic temperatures, inward conductive fluxes at the base of the corona, coronal areal expansion factors, outflow velocities (measured in the corona on the basis of either Doppler effect and Doppler dimming or radio scintillations), particle flux and the velocity of the associated wind streams in the heliosphere, as derived from the observations gathered in the pre-SOHO era. These parameters were used as physical constraints to develop a model, inclusive of radiative and conductive losses in the low corona and transition region, for four different types of solar regions: an equatorial coronal hole, a polar coronal hole at solar maximum and minimum and a unstructured quiet region of the corona (Withbroe 1988). Indeed, coronal holes display different characteristics during the solar cycle. For instance, polar coronal holes at solar maximum and equatorial coronal holes are significantly denser, by a factor of $2-3$, than polar coronal holes at solar minimum.

A study of the evolution of coronal holes carried out on the basis of He I $1083.0 \mathrm{~nm}$ emission, detected on ground during cycles 22 and 23, highlighted the fact that holes are very long-lived features with lifetime that can exceed 8 years. Typically, they originate in the two hemispheres at different times, develop initially at $50^{\circ}-60^{\circ}$ in latitude and within few solar rotations migrate to the poles (Harvey and Recely 2002). In the last two solar cycles, namely 23 and 24, there was the great opportunity to monitor continuously the evolution of coronal holes from space, measuring the EUV emission of the corona and the photospheric magnetic field - needed to infer the coronal field topology - with the SOHO, SDO, and STEREO instrumentation.

Coronal holes were concentrated in the polar regions over periods of several years including solar minimum, that is, from 1996 to 1998 and from 2006 to 2012. When the level of activity raised, polar holes shrank and, in turn, low-latitude holes became the source of a significant amount of the total open flux. The hemispheric field reversal, observed as a change of the polar coronal holes dominant polarity, was not synchronous in the two hemispheres (Lowder et al. 2017).

During the year 1996, except for a short period when a long narrow extension formed from the northern polar hole toward the equator (Del Zanna and Bromage 1999) similar to the Skylab CH1 configuration, low latitude coronal holes were almost absent. In these quiet Sun conditions, also the slow wind measured on the ecliptic plane was mainly originating from the two large polar holes, in their peripheral regions between the core, source of fast wind, and the boundary with the streamer belt (see Sect. 3.2). However, the scenario was quite different during the anomalous, prolonged and very weak minimum occurring at the end of cycle 23. Low-latitude holes, characteristic of the declining phase, remained a significant source of solar wind until the end of 2008, producing fast recurrent solar wind streams. At the end of 2008, these large low latitude coronal holes started to close down and, when they disappeared, small short-lived holes, not significantly contributing to the solar wind, formed at mid latitudes in the remnants of the first active regions of the new cycle (de Toma 2011), and as the cycle progressed, these mid-latitude coronal holes migrated toward the equator following the migration of activity bands (McIntosh et al. 2014).

According to the tendency of the global magnetic field to decrease from cycle 21 to cycle 24 (e.g., Bilenko 2018), during the quiet period from 2006 to 2009 the photospheric 
magnetic flux was indeed reduced relative to the previous cycle. In addition, a significant decrease in the area of the polar coronal holes, in comparison to the 1996 minimum, was also observed. The northern and the southern polar holes were, respectively, about $40-45 \%$ and 15-25\% smaller than in the previous minimum (de Toma 2011). At the end of the Ulysses mission in 2008, during the solar minimum of cycle 23 characterized by slower, less dense, and cooler solar wind, the wind emerging from polar coronal holes was weaker, with a density $20 \%$ lower and an electron component cooler by $15 \%$, whilst the current sheet retained a higher inclination (McComas et al. 2008). A statistical study of the low-latitude coronal holes observed during cycle 24 , excluding the equatorial extensions of polar holes, showed that the open magnetic flux is predominantly concentrated in flux tubes with high field strength (Hofmeister et al. 2017).

\subsection{Coronal Wind}

\subsubsection{Plasma Outflows in Coronal Holes}

Both the coronal configuration dictated by the magnetic field extrapolations together with the coronal emission measurements, and the theoretical modelling of the corona based on such observations defined a plausible scenario for the expansion of the solar corona. On the one hand, the open nature and areal divergence of magnetic fields in coronal holes were recognized as playing a crucial role in the acceleration and fast expansion of the coronal plasma (Kopp and Holzer 1976; Levine et al. 1977). Differences in physical parameters of the coronal regions, such as densities, temperatures and speed of the wind outflow, were related to differences in the configuration of the coronal magnetic field (Withbroe 1988). On the other hand, the solar minimum configuration of the large-scale magnetic field in the outer corona, with a predominant dipolar component, and the observations of the fast solar wind in the heliosphere obtained with SWICS (Gloeckler et al. 1992) on board Ulysses (Woch et al. 1997; McComas et al. 1998), implied that at solar minimum the high-speed plasma outflows emerging from coronal holes fill most of the solar corona. In addition, the measurement of the charge state of the oxygen ions in the heliospheric plasma, showed that the fast wind streams from polar holes were affected by slightly different temperatures, with the southern polar hole hotter by almost $10 \%$, with freeze-in temperature of $1.1 \mathrm{MK}$ (von Steiger et al. 2000; Posner et al. 2001).

Direct measurements of the dynamic conditions of the solar corona at the altitudes where the outflowing plasma is accelerated, however, became possible only during the SOHO mission, thus allowing us to progress in unambiguously identifying the sources and acceleration regions of the solar wind. These measurements were performed thanks to the introduction of spectroscopic observations of the resonantly scattered ultraviolet light emitted from the outer regions of the corona. The spectroscopic technique adopted in the analysis of the UV lines is based on the Doppler dimming effect, namely, a decrease in the emission of resonantly scattered lines, whose intensity depends on the relative velocity of the atoms/ions, present in the outflowing plasma and performing the scattering, and the source of the scattered photons originating at the base of the corona (Withbroe et al. 1982; Noci et al. 1987). The radial outflows of hydrogen and oxygen, the first and third most abundant elements in the Sun, have been thus traced in the corona by applying the Doppler dimming diagnostics to the resonantly scattered H I Lyman- $\alpha$ line and O VI doublet, measured with UVCS. This technique is sensitive to outflow velocities of oxygen ions and neutral hydrogen/protons above $\sim 20 \mathrm{~km} \mathrm{~s}^{-1}$ and $\sim 80 \mathrm{~km} \mathrm{~s}^{-1}$, respectively. 
Fig. 6 Composite image showing the wind outflow velocity derived via Doppler-dimming from UVCS O VI synoptic data acquired between August 19 and September 1,1996 , at $2.5 R_{\odot}$ for different orientations of the UVCS slit in the corona (filled squares) and the magnetic field lines configuration extrapolated by means of a 3D MHD model from the Kitt Peak National Observatory disk magnetogram obtained on August 19, 1996 (courtesy of Z. Mikić, Predictive Science Inc.). The fast wind is observed along the divergent open field lines emerging from the core of the polar coronal holes, whereas the slow wind is channeled along field lines emerging in the peripheral regions close to the boundaries of coronal holes

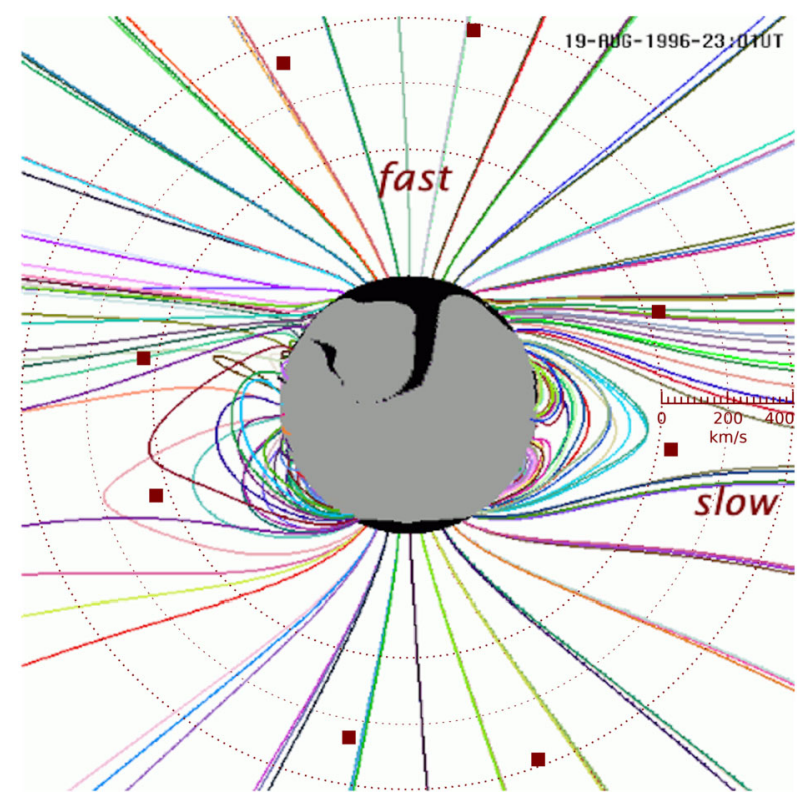

Up to now, since most of the spectroscopic observations of coronal wind outflows are extending only out to a few solar radii from Sun center, the wind speed can be measured in regions of the corona where the influence of the magnetic field on the plasma remains dominant. That is, outflows are traced not much beyond the radius of the source surface assumed, in the potential magnetic field extrapolations, to correspond to the height where coronal plasma is no longer confined and all lines of force of the magnetic field dragged by the wind open up nearly in a radial direction. Magnetohydrodynamic simulations of the solar corona, however, show that the influence of the magnetic field geometry on the solar wind properties remains significant well beyond the source surface (e.g., Réville and Brun 2017).

The study of the dynamics of the quiet corona is facilitated by the presence of a simplified magnetic configuration and an almost negligible emergence of active regions. In these conditions, direct UVCS observations of the coronal wind show that the plasma flows more rapidly in the core of large polar coronal holes along open magnetic fields with smaller areal divergence, undergoing a quite rapid acceleration beyond $1.5-2 R_{\odot}$. Whereas, in the equatorial streamer belt, outflows are detected only beyond a few solar radii from Sun center and they are characterized by very low speeds (Fig. 6). Where the field lines channel the fast wind, oxygen ions, neutral hydrogen and protons flow roughly at the same velocity approximately out to $\sim 3 R_{\odot}$ (e.g., Antonucci et al. 2000). Due to the low density of the coronal hole plasma, protons decouple from neutral hydrogen atoms (e.g., Olsen et al. 1994; Allen et al. 1998) approximately at a height of $10 R_{\odot}$, thus the wind proton component in principle could be observed out to this level; however, at about $4 R_{\odot}$ the H I Lyman- $\alpha$ emission of the corona is predicted to become less intense than the interplanetary Lyman- $\alpha$ emission (Spadaro et al. 2017). Thus, in the core of coronal holes the Doppler dimming analysis allows us to track the flow of the neutral hydrogen and proton wind components only as far out as $\sim 4 R_{\odot}$. The oxygen component, on the other hand, can be traced further out, from $\sim 40 \mathrm{~km} \mathrm{~s}^{-1}$ at $1.5 R_{\odot}$ (Kohl et al. 1998; Antonucci 1999; Cranmer et al. 1999a; Antonucci et al. 2000) all the way to speeds in the range $550-760 \mathrm{~km} \mathrm{~s}^{-1}$, at $5 R_{\odot}$ 
(Telloni et al. 2007a), which are approaching the asymptotic wind values measured in the heliosphere $\left(\sim 800 \mathrm{~km} \mathrm{~s}^{-1}\right)$. Thus, only the oxygen ions can be studied throughout most of their acceleration region based on the UVCS observations.

According to the most plausible interpretation, the remarkable acceleration of the oxygen component in the core of coronal holes is due to energy dissipated by ion-cyclotron resonant Alfvén waves in the outer corona (Cranmer et al. 1999b, 2017). By measuring the extremely large kinetic temperature observed in coronal holes, which represents the distribution of both thermal and non-thermal velocities of the oxygen ions along the line of sight approximately perpendicular to the field lines, we can deduce that energy is dissipated across the magnetic field at a maximum rate around $2.9 R_{\odot}$ (Telloni et al. 2007b). Namely, beyond the sonic point that, according to a statistical analysis of the UV spectroscopic observations of coronal holes throughout cycle 23 , is located around $1.9 R_{\odot}$ (Telloni et al. 2019). That is, the peak in energy release occurs in a region where its effect is that of increasing the wind speed (Holzer and Leer 1997).

\subsubsection{Outflow Speed and Magnetic Field Topology}

The spectroscopic observations with UVCS of the coronal wind during solar minimum provided direct evidence for a correlation of coronal plasma parameters with the geometry of the flux tubes channeling the wind outflows. In addition, these observations showed that the magnetic field expansion factor is not only correlated with the speed of the outflowing plasma, but also with other physical properties, such as, the velocity distribution across the magnetic field of the atoms/ions emitting ultraviolet photons by resonant scattering and the plasma elemental composition in corona.

In the northern hemisphere of the corona observed during the Skylab period and studied by Munro and Jackson (1977), approximately $60 \%$ of the entire atmosphere above $3 R_{\odot}$ was found to be connected to about $8 \%$ of the solar surface, that is, in polar coronal holes magnetic field lines were found to be super-radial, and the increase of the polar hole's crosssectional area from the surface to $3 R_{\odot}$ turned out to be about 7 times greater than if the boundary were purely radial.

The divergence of the field lines increases moving away from the core to the periphery of the coronal hole (Wang and Sheeley 1990) to reach a maximum value close to the streamer boundary (Dobrzycka et al. 1999), where the expansion factor of the flux tubes can be even a factor 3 higher than in the hole center (Abbo et al. 2010). As the areal divergence of the flux tube increases the outflow speed decreases, giving origin to a slower wind. A clear example of the interdependence of these two quantities is given, for instance, by Cohen (2015), who traces the field geometry and the outflow speed in the corona during the minimum of cycle 22 with his magnetohydrodynamic model. In addition, the coronal hole flux tubes lying in the vicinity of the streamer boundaries show a characteristic non-monotonic expansion before the coronal wind reaches the critical point, where the flow becomes supersonic.

As an example, the magnetic field lines rooted just above $-63^{\circ}$ latitude at the edge of the southern coronal hole observed at the end of August 1996, outlining a region of nonmonotonic expansion, diverge rapidly to reach the maximum expansion at about $2.5 R_{\odot}$, and then they converge to $\geq-14^{\circ}$ latitude at $6 R_{\odot}$; on the other hand, field lines, emerging from the core of a coronal hole above approximately $-70^{\circ}$ latitude, diverge monotonically. A similar non-monotonic flux tube configuration also occurs in the case of the field lines separating sub-streamers (Fig. 5) in the complex structures observed at solar minimum close to the equator (see review by Antonucci et al. 2012, and references therein).

The images of large quiescent equatorial streamers obtained with UVCS during 1996 showed a striking difference in $\mathrm{H}$ I and O VI emission (Fig. 7). A strong dimming was 
Fig. 7 H I Lyman- $\alpha$ and O VI $103.2 \mathrm{~nm}$ images of the 10 August 1996 equatorial streamer observed between $1.5 R_{\odot}$ and $3 R_{\odot}$ with UVCS at the east limb. The O VI streamer consists of bright sub-structures converging toward the interplanetary current sheet beyond $2.5-3 R_{\odot}$

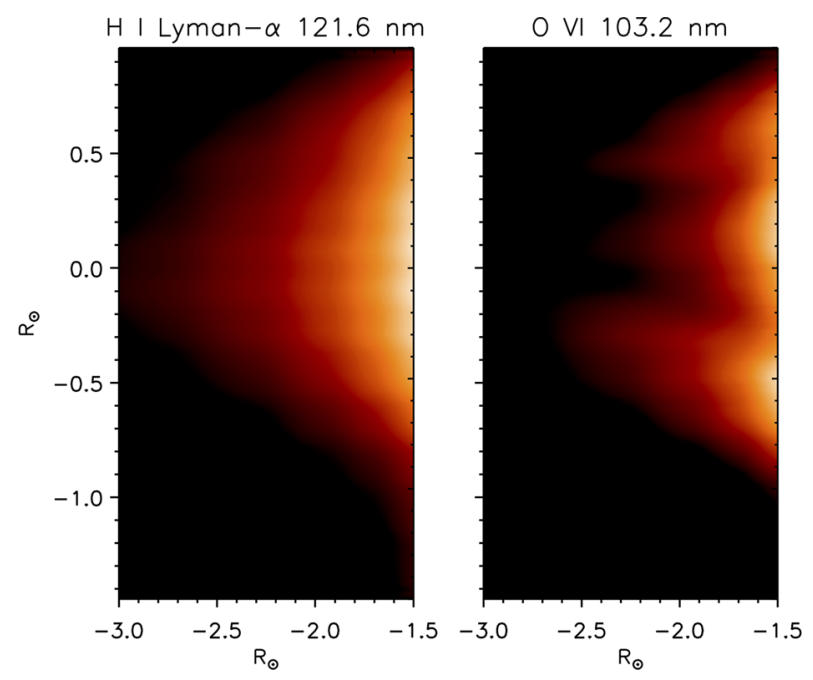

detected in the core of the streamer belt but only in the case of the O VI lines. This peculiar morphology of the oxygen emission revealed a local depletion of oxygen of roughly one order of magnitude with respect to the photosphere. Noci et al. (1997) suggested that this effect is due to the geometry of fluxtubes of open magnetic field lines that separate the sub-streamers in the equatorial belt.

Simple streamers formed by one closed loop system may show an attenuated emission from their core as a consequence of projection effects (Abbo et al. 2019); in this case, however, the effect is not related to the formation of the slow wind since this requires the existence of multiple loop structures within the streamer to give origin to outflows emerging directly from the streamer core. The core dimming signature is lacking in active streamers that may be present in the streamer belt (Uzzo et al. 2003) as well as in active region streamers, although exceptions can be found (Uzzo et al. 2004).

According to Noci et al. (1997), oxygen core dimming can be explained as follows. The flux tubes guiding outflows of coronal plasma between sub-streamers are characterized by a variable cross-section: an initially high areal divergence is followed by a narrowing of the fluxtube before reaching the critical point where the wind speed exceeds the sound speed (Fig. 8). This geometry has the effect of slowing down the wind relative to a radial flow. The effect on the proton and oxygen velocity of the flux tube expansion factor, increasing rapidly in the inner corona and then decreasing further out, has been modeled for instance by Chen et al. (2004).

A wind speed decrease with unchanged density implies a reduced proton flux. Since the oxygen ions are dragged into the solar wind via Coulomb collisions by a force proportional to the proton flux (Geiss et al. 1970), a reduction in this flux causes a decrease of the oxygen abundance, which is observed as an O VI dimming in the streamer core. This interpretation is consistent with the presence of very weak outflows in the core of complex streamers, with a speed $\sim 20 \mathrm{~km} \mathrm{~s}^{-1}$ at $2.2 R_{\odot}$ (Noci and Gavryuseva 2007, see Fig. 8), which is much lower than the velocity of the fast wind measured at about the same height, exceeding $200 \mathrm{~km} \mathrm{~s}^{-1}$ (Kohl et al. 1998; Cranmer et al. 1999a; Antonucci 1999; Antonucci et al. 2000). Moving outward along the streamer axis, the wind accelerates at a low rate to reach a value close to $100 \mathrm{~km} \mathrm{~s}^{-1}$, approximately in the interval 3.5-5 $R_{\odot}$ (Habbal et al. 1997; Strachan et al. 2002; Antonucci et al. 2005; Susino et al. 2008; Abbo et al. 2010). 
Fig. 8 Sketch of the magnetic topology of a complex streamer formed by loop systems separated by fluxtubes with non-monotonic expansion factor. Arrows show the slow solar wind flow. A map of the O VI emission, degrading toward higher heliodistance, is superposed as an example; the red curve indicates the position of the minimum of the O VI 103.7, $103.2 \mathrm{~nm}$ doublet intensity ratio corresponding to an outward flow of $\sim 20 \mathrm{~km} \mathrm{~s}^{-1}$, as derived from a (west) limb streamer observed on August 26, 1996 (readapted from Noci et al. 1997; Noci and Gavryuseva 2007)

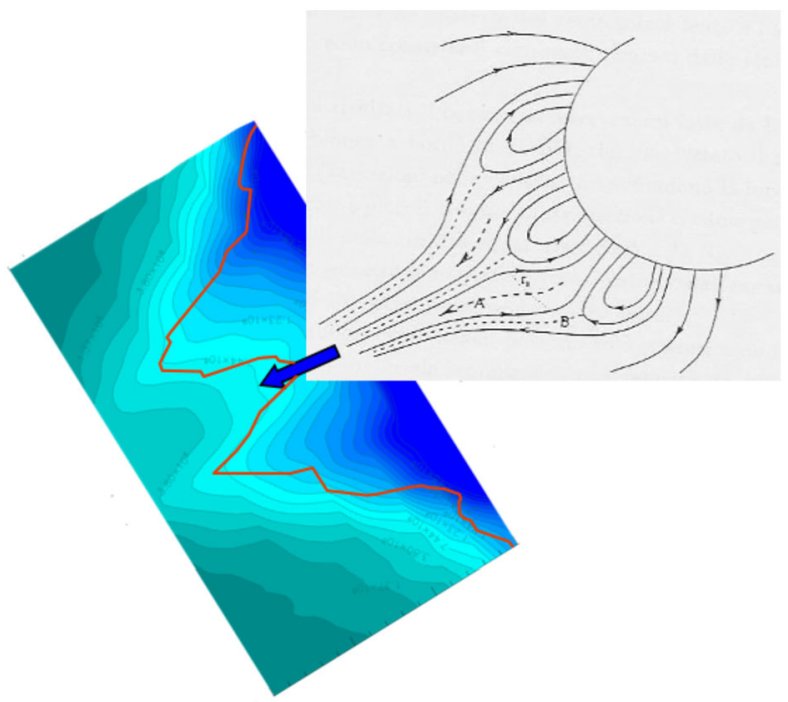

The coronal hole regions adjacent to the streamer boundaries are characterized by the same magnetic topology inferred for the lanes dividing sub-streamers in the equatorial belt. In these hole boundary layers at a height close to $\sim 2 R_{\odot}$, the slow wind flows faster $\left(\sim 100 \mathrm{~km} \mathrm{~s}^{-1}\right)$ than that observed along the streamer axis (Noci and Gavryuseva 2007). In particular, Strachan et al. (2002) measures a wind speed of $\sim 100 \mathrm{~km} \mathrm{~s}^{-1}$ at $2.3 R_{\odot}$, and on the basis of an analysis of a sample of six streamers and their surrounding regions observed during solar minimum, Antonucci et al. (2005) measure speeds ranging from $\sim 90 \mathrm{~km} \mathrm{~s}^{-1}$ at $1.8 R_{\odot}$ to $\sim 110 \mathrm{~km} \mathrm{~s}^{-1}$ at $3.5 R_{\odot}$. Values of the same order are obtained by Spadaro et al. (2005) and Abbo et al. (2010). In the region where the spectroscopic observations of the corona can still be performed, out to approximately $5 R_{\odot}$, the slow wind does not yet reach its asymptotic value $\left(\sim 400 \mathrm{~km} \mathrm{~s}^{-1}\right)$. Therefore, the slow wind is undergoing acceleration processes also beyond $5 R_{\odot}$.

Summarizing the above results, the UVCS observations of the quiet corona hence suggest that the slow wind essentially consists of two components. In addition to the slow wind flowing along the flanks of coronal holes, there is a minor component of very slow wind emerging from the core of the magnetically complex streamers formed by an odd number of sub-streamers. The dual behavior of the slow wind is observed both for the oxygen ions (Antonucci et al. 2005) and for hydrogen atoms, whose acceleration, however, is relatively more gradual (Susino et al. 2008).

Wang et al. (2000) reached the same conclusion about the existence of a two component slow wind by studying the flow of small plasma inhomogeneities, or coronal blobs, forming at the streamer cusp and moving along the current sheet (Sheeley et al. 1997; Wang et al. 1998). The speed of coronal blobs is indeed consistent with that of the slow wind deduced by Doppler dimming techniques just above the streamer cusp. These features, however, constitute a small fraction of the slow solar wind, e.g. $15 \%$ at solar maximum (Sanchez-Diaz et al. 2017). Observed with the Heliospheric Imager of STEREO throughout a solar cycle (Plotnikov et al. 2016), blobs tend to occur in a quasi-periodic manner, presumably as a result of an intermittent reconnection process (Sanchez-Diaz et al. 2017).

The coronal outflow speed has also been derived by applying Fourier motion filters to SOHO/LASCO C3 movies of the white light emission of the outer corona observed from 
6 to $26 R_{\odot}$, in the years $2006-2010$ during the minimum of cycle 23 . This analysis adds further observational evidence for the presence in the corona of the polar fast wind and slow outflows toward the equator. This bi-modal configuration of the wind disappears at the beginning of the new solar cycle (Cho et al. 2018). The fact that both fast and slow wind are emanating from coronal holes, is also inferred by tracing back to the solar surface the heliospheric wind measured in situ (Zhao et al. 2017).

By identifying the heliospheric slow wind as the flow associated with a regime of low velocity fluctuations, Ko et al. (2018) suggests that in addition to the slow wind observed in the region of super-radial expansion of open coronal magnetic field at the boundary of the coronal hole and the very slow wind along the streamer axis, there is a further component originating in the interface region where interchange reconnection between the coronal hole open field and the adjacent closed loops is likely to occur. Both this further layer and the streamer axis region are suggested to be the site where episodic slow wind is originating in reconnection events.

The direct observations during solar minimum of the dynamics of the neutral hydrogen and oxygen components in the outer corona confirm the expected latitudinal distribution of the fast and slow solar wind from the poles to the low-latitude regions, highlight a larger variability characterizing the slow wind speed, and show a hemispheric wind asymmetry, being the polar wind speeds in the northern hemisphere systematically higher than those in the southern regions, with a degree of asymmetry which tends to decrease with heliospheric distance (Dolei et al. 2018). The fast wind is the dominant dynamic coronal component, since the slow wind is predominantly confined in the coronal hole peripheral regions and, further out, along the heliospheric current sheet, with an additional although minor contribution of slow wind emerging from the field lines separating sub-streamers or associated with reconnection events at the streamer cusp and along the streamer-coronal hole interface. These observational results, hence, confirm the conclusions reached by Levine et al. (1977), Wang and Sheeley (1990), and Bravo and Stewart (1997), concerning the existence of a correlation of magnetic topology and the speed of the coronal wind flowing more slowly along magnetic flux tubes with larger expansion factors and they are fully consistent with the fact that the rate of flux tube expansion is inversely correlated with the solar wind speed at $1 \mathrm{AU}$ (Wang et al. 2007; Abbo et al. 2016). Moreover, the UVCS observations have highlighted the importance of non-monotonically diverging fluxtubes in controlling the wind speed.

Panasenco and Velli (2013) modeled the areal expansion factor in order to infer the wind speed along non-monotonically diverging field lines in the vicinity of pseudo-streamers during quiet, or relatively quiet, coronal conditions. The reconstructed magnetic field configuration reveals that the geometry of the flux-tubes determining the wind speed is more complex than that associated with the polar coronal hole boundary regions. The study of the coronal outflows in the vicinity of a pseudo-streamer by Abbo et al. (2015) suggests that it is unlikely that pseudo-streamers and their immediate surroundings are sources of slow solar wind (Wang 2012; Wang et al. 2012).

Evidence for Energy Deposition in the Slow Wind The magnetic field divergence is not only related to the wind speed but also to the velocity distribution of atoms/ions in the outer corona, that is interpreted as a signature of the local energy deposition across the magnetic field through ion-cyclotron resonance of high-frequency Alfvén waves (Kohl et al. 1998; Cranmer et al. 1999a,b). The early EUV and X-ray observations of the inner corona revealed a strong correlation between the structure of the solar magnetic field and plasma heating in the solar atmosphere, since the energy flux is guided by the magnetic field that controls the geometry of coronal features (Withbroe and Noyes 1977). Recently, Cranmer 
et al. (2017) have summarized the behavior of wind streams flowing along flux tubes with different expansion factors, as derived from the direct measurements in corona obtained with the SOHO instrumentation.

Observationally, fast and slow streams differ in terms of radial distribution of the heating rate and location of the critical point. Kinetic temperatures, inferred from the broadening of the spectral lines due to the distribution of thermal and non-thermal velocity of the oxygen ions along the line of sight, that is approximately across the coronal magnetic field in measurements at the limb, are observed to decrease from the extremely large values attained in the core of coronal holes to lower values moving toward the streamer boundary (Antonucci et al. 1997). For instance, at a height $\sim 3 R_{\odot}$, the kinetic temperatures of the oxygen ions, which in the polar regions are of the order of $10^{8} \mathrm{~K}$ (e.g., Telloni et al. 2007b), decrease to typical values of the order of $10^{7} \mathrm{~K}$ in the periphery of coronal holes where the slow wind flows (Strachan et al. 2002; Antonucci et al. 2005; Spadaro et al. 2005; Susino et al. 2008; Abbo et al. 2010). These values are still one order of magnitude higher than the electron temperature. Hence, observations show that energy is also deposited, although at a different rate, in the slow wind regions.

The decrease of kinetic temperatures with increasing expansion factor points to a more effective energy deposition in the less divergent magnetic fields, and a flux and rate of dissipation of Alfvén waves depending on the field geometry. Furthermore, in the fast wind, energy is clearly observed to be predominantly deposited beyond the critical point, thus increasing the outflow speed to a fast wind regime. In the slower wind, signatures of energy deposition are evident below the sonic point (Antonucci 2006), thus the main effect is that of increasing the mass flux of the outflowing plasma (e.g., Holzer and Leer 1997).

Elemental Abundances in the Coronal Wind Although the elemental composition measurements in the corona based on the oxygen abundance are affected by a non-negligible degree of uncertainty due to the assumptions involved in the data analysis, a comparison of coronal and heliospheric composition inferred from the Ulysses data is consistent with the identification of the coronal sources of the fast and slow wind with the core and flanks of coronal holes, respectively, as discussed in the previous sections. The oxygen abundance relative to hydrogen, $6.0 \times 10^{-4}$, measured with UVCS in the fast wind at coronal level (Antonucci and Giordano 2001) retains substantially the photospheric value, $6.7 \times 10^{-4}$ (at least according to earlier spectroscopic results; see Grevesse and Sauval 1998), and is close to the value detected as a function of wind speed in the interplanetary space for high-speed streams, $\sim 6.3 \times 10^{-4}$, by von Steiger et al. (1995). According to a thorough study encompassing the entire Ulysses mission, in the fast wind streams identified on the basis of the freeze-in temperature as inferred from the $\mathrm{O}^{7+} / \mathrm{O}^{6+}$ measured with SWICS, von Steiger et al. (2010) re-assessed the value of the oxygen abundance, $6.6 \times 10^{-4}$, which can be assumed to be the solar oxygen abundance. The authors discuss the oxygen abundance derived from the Ulysses data in comparison to that derived from spectroscopic results, re-evaluated toward progressively lower values, and its important implications in terms of solar models based on helioseismology results.

Along the flanks of coronal holes above $2 R_{\odot}$ the oxygen abundance - although affected by the assumptions to be made in data analysis (Antonucci 2006) - has a lower value consistent with the heliospheric slow wind condition, $5.3 \times 10^{-4}$. The flows emerging from the core of the quiescent streamers observed with UVCS (Marocchi et al. 2001) are instead particularly poor in oxygen compared with the heliospheric slow speed streams. At $1.7-2.2 R_{\odot}$ the oxygen abundance in the streamer core drops to about $1.0 \times 10^{-4}$, implying that the flow emerging from the core is indeed a minor component of the slow wind. This value is well 
within the upper limit of oxygen abundance, $\geq 4.0 \times 10^{-4}$, measured during the Ulysses passages across the heliospheric current sheet in the phase of declining activity of cycle 22 (von Steiger et al. 1995).

In 2009, during the solar minimum of cycle 23, the first measurements of He II $30.4 \mathrm{~nm}$ line intensity, combined with simultaneous measurement of the $\mathrm{H}$ I line, were obtained over the west limb of the Sun out to $2.2 R_{\odot}$ with the Sounding-rocket CORonagraph Experiment (SCORE), during the first flight of the HElium Resonance Scattering in the Corona and HELiosphere (HERSCHEL) sounding rocket (Moses et al. 2020). This investigation led to the conclusion that, as in the case of oxygen, the helium abundance in the outer corona is influenced by the topology of the magnetic field lines. Very low values are attained in the equatorial region: values $\leq 3 \%$ are observed within $\pm 15^{\circ}$ across the equator, with a minimum value, $0.6 \%$, close to the equator. Outside this region, the He abundance progressively increases although retaining a value $\leq 10 \%$, limit that is close to the outer convection zone abundance. The extremely low abundance of the helium ions in the coronal equatorial region is suggested to cause the anomalously low helium abundance, $<0.5 \%$, measured at the same time with the Wind spacecraft instrumentation in the heliosphere, at L1, in the slow wind flowing at $260 \mathrm{~km} \mathrm{~s}^{-1}$ (Kasper et al. 2012).

The comparison of coronal and heliospheric abundance data support the interpretation that the main contribution to the slow wind comes from the boundary layers of coronal holes, where the outflowing plasma is dynamically fractionated because of the low proton fluxes associated with the large geometrical expansion factors of the flow tubes surrounding coronal streamers.

\subsubsection{Evolution of the Open Corona in the Rising Phase of Solar Activity}

Strachan et al. (2012) analyzed the evolution of the outflow velocity and density of the corona, combining the UVCS and LASCO data, in order to measure the expansion of the solar wind from the coronal base to the source surface height, set at $2.3 R_{\odot}$, during the transition from the solar minimum of cycle 22 to the rising phase of cycle 23 , in the period from 1996 to 1998 . When the activity rises and the polar holes shrink, Strachan et al. (2012) observe changes not only in the global area of the coronal hole but also in the areal divergence of the flux tubes channeling the solar wind. The very large expansion factors of the flux tubes observed near the streamer belt during solar minimum tend to vanish when the overall size of the polar holes decreases. According to Strachan et al. (2012), larger coronal holes have the largest areal expansion and emanate the fastest solar wind. This result is consistent with the finding that there is a correlation of the area of coronal holes and the solar wind speed (Zhao et al. 2017; Tokumaru et al. 2017).

Selecting large low-latitude coronal holes, Miralles et al. (2001) and Miralles et al. (2004) show that although at $3 R_{\odot}$ the related coronal outflow speed is significantly lower than in polar holes, the associated heliospheric high-speed streams reach anyway high asymptotic velocities of $600-750 \mathrm{~km} \mathrm{~s}^{-1}$. This correlation might imply that at solar maximum in low latitudes holes the bulk of the solar wind acceleration is likely to occur above $3 R_{\odot}$.

Heliospheric observations suggest the existence of a sharper interface boundary between fast and slow wind streams than in the corona. All physical parameters, such as speed, charge-state composition, density, elemental composition, tend to change quite abruptly between the two wind regimes (e.g., Schwenn 2000; von Steiger et al. 2000). On the other hand, the UVCS results on the dynamics of the solar corona indicate that an abrupt variation, from expanding to static coronal plasma, occurs just at the streamer-hole interface between closed and open field lines. The transition from fast to slow wind occurs gradually within a 
relatively narrow region, approximately $20^{\circ}$ wide, at each side of the coronal current sheet (Habbal et al. 2001; Antonucci et al. 2005; Susino et al. 2008). The slow wind in the heliosphere as observed by Ulysses fills a cone of about $\pm 21^{\circ}$ with the symmetry axis tilted about $7^{\circ}$ to $8^{\circ}$ with respect to the heliographic equator, and continuous observations of the slow solar wind through a solar rotation, without any encounter of fast solar wind streams, are restricted to a more limited region with a width of about $13^{\circ}$ around the equator (Woch et al. 1997). This would imply that, since the available observations of the solar corona are more in favor of a relatively gradual distribution of wind speeds than the bi-modal distribution observed in the heliosphere, a sharper discontinuity as measured with in situ instruments develops during the propagation of the coronal wind toward the heliosphere.

In this section, we have discussed from the observational point of view the magnetic topology of the corona and its connection with the solar wind primarily during minimum of activity, when fast and slow solar wind are well-defined and when the flux tube expansion is the key regulator of the wind speed, with particular emphasis on the non-monotonic expansion of flux tubes, giving origin to the slowest wind. Considering solar wind throughout the various solar conditions, Antiochos et al. (2012) introduce the complementary concept of quasi-steady, whether fast or slow, and non-steady, always slow, winds, and discuss the different hypotheses to explain the non-steady slow wind not only based on the expansion factor which however appears to be sufficient to explain the main features of solar minimum wind conditions.

\subsubsection{Waves and Oscillations in the Coronal Wind}

The role of magneto-hydrodynamic waves and oscillations in the solar corona is crucial not only in the heating processes of the coronal plasma, as mentioned in Sect. 2.1, but also in accelerating the solar wind, as indicated by evidence for Alfvén waves found in the acceleration region of the solar wind, inferred from non-thermal line broadening effects in the resonantly emitted line profiles observed with UVCS (e.g., Cranmer et al. 1999a), as reported in Sect. 3.2.2. Fluctuations and oscillations of the plasma density in the solar corona are observed over a wide range of periodicities, from few minutes to several hours, out to at least $3 R_{\odot}$ (e.g., see the review by Nakariakov and Verwichte 2005).

Large-scale density fluctuations with periods spanning from a few hours to a few days have been identified in the outer corona, both in polar (Bemporad et al. 2008; Telloni et al. 2009a) and equatorial (Telloni et al. 2009b) regions, based on UVCS observations. Whether the density fluctuations observed in the slow wind of the solar corona are due to plasma inhomogeneities advected by the expanding coronal plasma or they are to be ascribed either to upward propagating magneto-acoustic waves or to non-propagating discontinuities separating spatial structures with different density characteristics (as for instance magnetic flux tubes) and crossing the field of view of the spectrometer during a solar rotation, is not yet fully understood.

The spectra of density fluctuations differ from those of the interplanetary magnetic field that exhibits a $1 / \mathrm{f}$ dependence at low frequencies in high-speed streams because of the different nature of density and magnetic field fluctuations. Magnetic field fluctuations in the fast wind are due to non-compressible Alfvénic fluctuations, thus there exists a limiting value for the fluctuations represented by the magnetic magnitude, reached at frequencies lower than the correlation length. This sort of saturation reflects into a $1 / \mathrm{f}$ spectrum of the magnetic field Alfvénic fluctuations (see Matteini et al. 2018; Bruno et al. 2019, for more exhaustive explanations). This does not apply for density fluctuations, which are compressive in nature and, thus, are not limited by any particular value of fluctuations. It follows that the power spectrum of density fluctuations does not generally exhibit the 1/f power-law behavior. 
Fig. 9 Power density spectra (PDS) of the density fluctuations observed in the fast (top panel) and slow (bottom panel) wind at coronal and heliospheric heights (black and red curves, respectively); solid lines show the corresponding spectral scaling. Uncertainty bars correspond to a 95\% confidence level

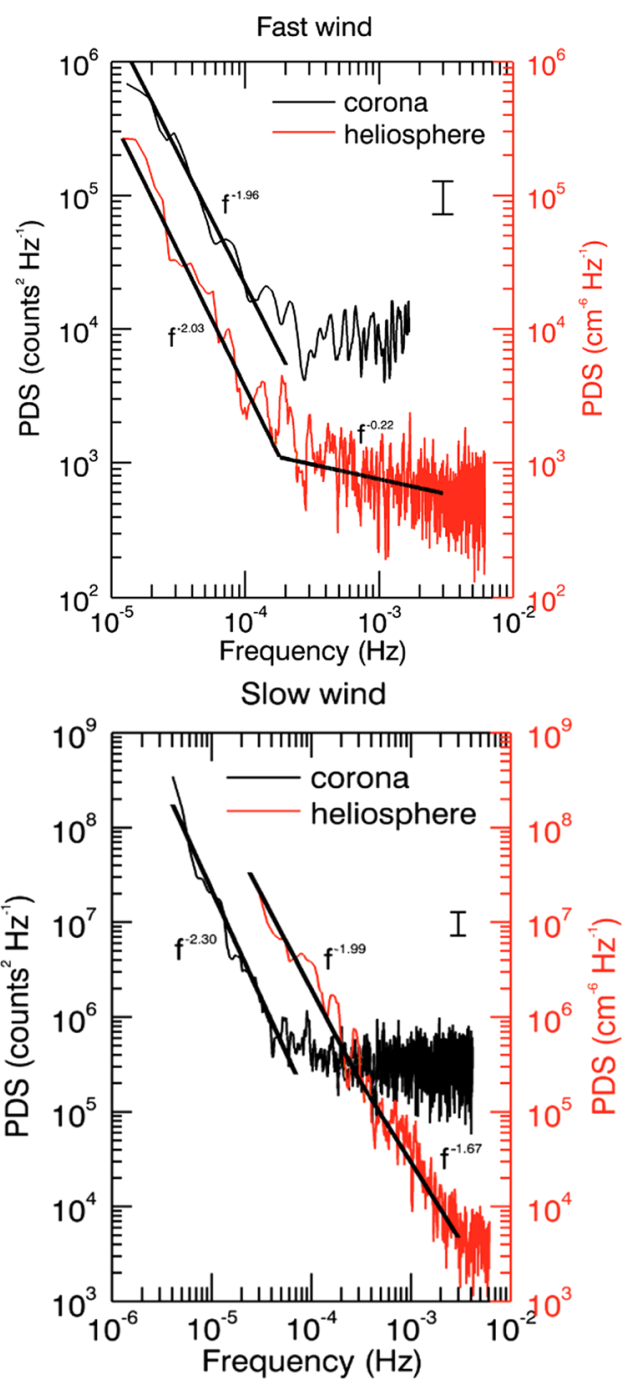

The evolution of the power spectra of the density fluctuations from coronal to heliospheric height is different for the slow and fast wind regimes. Slow wind fluctuations are dominated by persistent structures, coherent in phase for time scales larger than $10^{4} \mathrm{sec}$. When moving from the outer corona to the inner heliosphere, the spectral evolution indicates a change from persistent, correlated density fluctuations to temporally uncorrelated structures as occurs in the case of both the coronal and heliospheric fast wind (Fig. 9, top panel). The long-range correlations found at low frequency in the coronal spectrum of the slow wind can be interpreted as driving a turbulent cascade in the transition from the corona to the heliosphere, where the slope of the high frequency spectrum is observed to be Kolmogorov-like. The development of the turbulent cascade accumulates coherent structures at smaller scales, hence, in the slow wind the density fluctuation spectrum evolves quickly during the transition from the corona to the inner heliosphere (Telloni et al. 2009a). 
Fig. 10 2D map of the oscillating coronal density structures, in timescale band ranging from 4 and $8 \mathrm{~h}$, on April 18, 2008 at 21:50 UT, obtained from COR1-A observations; the SOHO MDI disk magnetogram as seen from STEREO-A viewpoint is also shown; the inset figure shows the extrapolated magnetic field lines

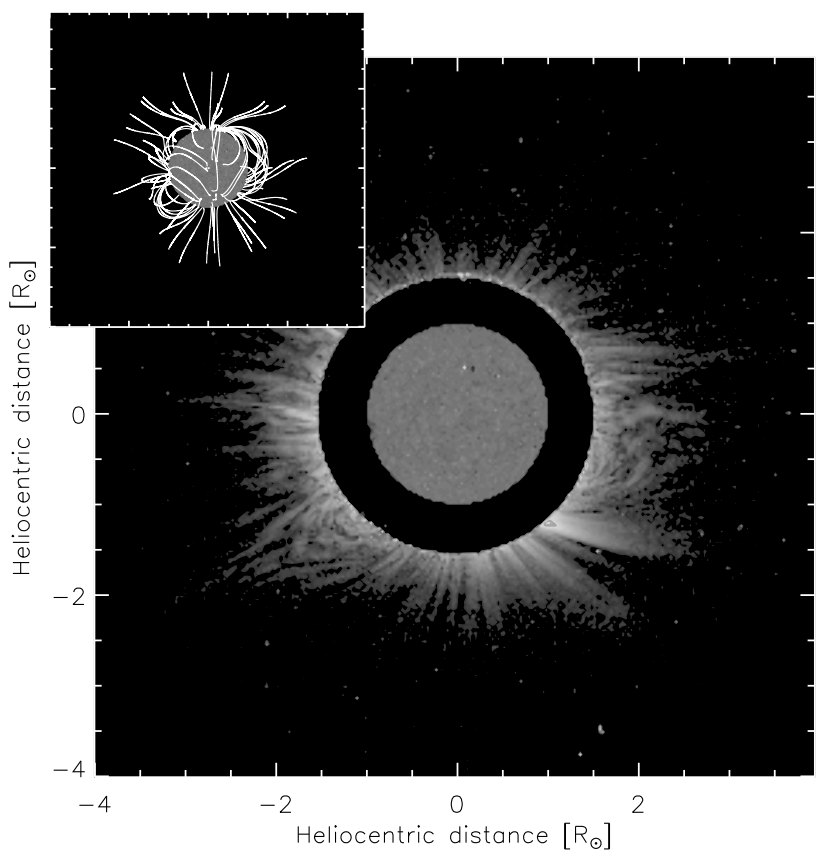

Furthermore, Telloni et al. $(2013,2014)$ have studied the spatial and temporal variability of the oscillation amplitude of the coronal total brightness derived from a long-term series of white-light images acquired at high cadence by the STEREO COR1-A coronagraph (Howard et al. 2008). The image analysis, based on the wavelet decomposition of the observed time series into the time-frequency space and the $2 \mathrm{D}$ visualization of the temporal modulation of the oscillation power at a certain timescale, has shown that at least out to $3 R_{\odot}$ the solar corona is ubiquitously permeated by quasi-periodic recurrent plasma fluctuations, which encompass a wide range of spatial and temporal scales. However, these density oscillations are not uniformly distributed. They are instead observed to outline the magnetic field lines (Fig. 10). In polar regions, they assume the form of ray-like structures. It is interesting to note that the fluctuations embedded in coronal ray-like structures can be interpreted as signatures of either intermittent magnetic reconnection, for instance, due to continuously emerging flux tubes, or quasi-periodic transverse displacements of such features. The identification of these ray-like fluctuating features is at present uncertain also because their roots at the poles can be only observed with instruments located on the ecliptic plane and not yet imaged from an out of the ecliptic vantage point.

\subsection{Asymmetries in the Large-Scale Organization of the Solar Corona}

\subsubsection{Southward Displacement of the Heliospheric Current Sheet}

During the declining to minimum phase of the solar cycle, the open coronal magnetic field inferred on the basis of photospheric observations obtained at the Wilcox Solar Observatory from 1976 on, is constantly asymmetric with respect to the heliographic equator (Zhao et al. 2005; Virtanen and Mursula 2016; Getachew et al. 2017; Koskela et al. 2018). That is, the coronal current sheet exhibits a persistent shift of about $10^{\circ}$ toward the southern hemisphere. 
On the other hand, in the solar activity phases other than the declining and minimum phase, the displacement from the heliographic equator is quite insignificant (Zhao et al. 2005).

The hemispheric asymmetry in the open coronal magnetic field in turn induces a shift in the same direction, although of reduced extent, of the heliospheric current sheet derived from the interplanetary magnetic field polarity structure (Wilcox 1972; Smith et al. 2000; Mursula and Hiltula 2003; Wang and Robbrecht 2011; Koskela et al. 2018). It was noticed (Wilcox 1972; Antonucci 1974a) that in the years 1954 and 1965, during the solar minimum of the cycles 18 and 19, the interplanetary magnetic field inferred from high latitude geomagnetic field data (Svalgaard 1972) did not exhibit the characteristic sector structure polarity pattern, but maintained the same positive polarity for a few solar rotations. During 1954, the persistence of the field pointing outward from the Sun for more than 3 solar rotations produced an anomalous cosmic ray anisotropy (Antonucci 1974a). Hiltula and Mursula (2006) showed that the north-south asymmetry is a characteristic feature of the solar and heliospheric current sheets during the declining to minimum phase of the activity cycle, and, owing to the influence of the polarity of the heliospheric field on the geomagnetic data, this effect can be traced back at least to 1926. In this long-lasting interval, from cycle 16 to cycle 23, only cycle 19 represents an exception (Hiltula and Mursula 2006), and the shift of the neutral line in the corona, as measured from cycle 21 on, is larger than the shift of the heliospheric current sheet at $1 \mathrm{AU}$ (Koskela et al. 2018).

The southward shift of the heliospheric current sheet implies an asymmetry of the open solar magnetic field with respect to the equator. Wang (2014) notes that any hemispheric asymmetry in sunspot activity gives rise to an axis-symmetric quadrupole component which combines with the axial dipole to displace the current sheet in the north-south direction. On this basis, Wang relates the southward displacement of the coronal and heliospheric current sheet to the dominance of activity in the northern/southern hemisphere during the rising/declining phases in the time interval from cycle 20 to the initial phase of cycle 24 . When the activity in the northern hemisphere is the dominant one during the rising phase, as during cycles 20-24, the equatorial band of the magnetic quadrupole will have the same sign as the north polar field and the heliospheric current sheet will be shifted below the equator. On the other hand, when the activity in the southern hemisphere dominates during the declining phase, as during cycles 20-23, the equatorial band of the magnetic quadrupole will have the same sign as the leading polarity flux in the southern hemisphere, which is opposite to the sign of the south polar field, therefore the heliospheric current sheet will again be shifted below the equator. Cycle 19 displays an opposite behavior as in this cycle the northern activity was the predominant one in the declining phase.

The long-lasting period of observation of the southern displacement of the heliospheric current sheet includes intervals with different hemispheric phase of activity (Deng et al. 2016, and references therein). That is, during the most recent cycles, from 20 to 24 , the northern hemisphere was more active on the ascending branch, whereas the southern hemisphere, lagging in phase, dominates on the descending branch of the solar cycle. Whereas, the southern hemisphere was leading in phase in the preceding cycles: either cycles 16-19 according to McIntosh et al. (2013), or 17-19 according to Deng et al. (2016), who finds that the phase-leading hemisphere returns back to the identical hemisphere every 8 solar cycles (thus, cycles 20-24 reproduce the group of cycles 12-16). It is therefore puzzling that since 1926 the southern shift of the heliospheric current sheet remains almost invariant, except during cycle 19, although in the first 4 cycles of the 8 -solar cycle period the dominant hemisphere in ascending and descending phase of the cycle is the opposite than observed in the subsequent 4 solar cycles. In any case, as noted by Zhao et al. (2005), the asymmetry of the heliosphere about the equator has to be associated with the characteristics of the open field 

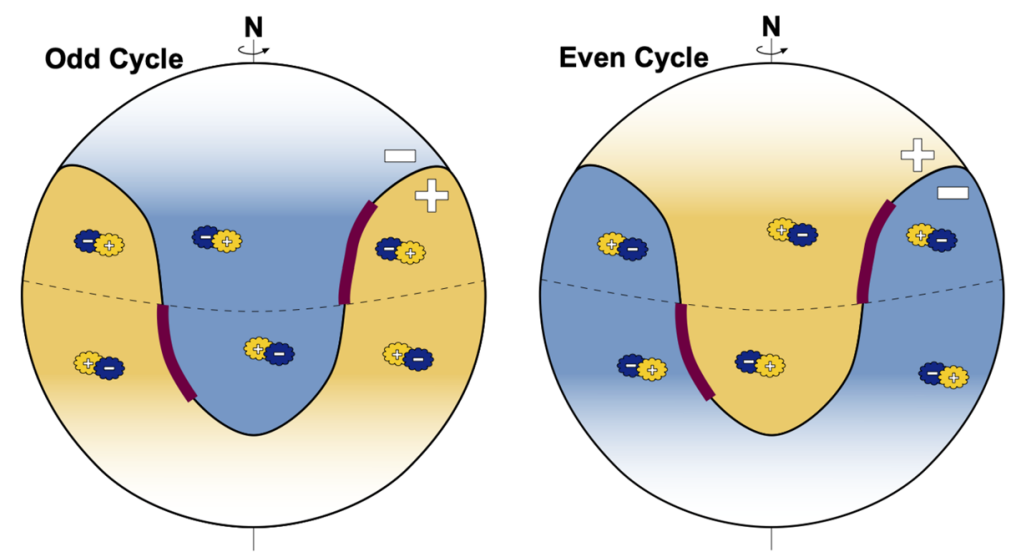

Fig. 11 Scheme of the Hale sector boundaries (purple line) for odd and even cycles when four polarity sectors are present in the magnetic field detected in the heliosphere. Hale boundaries indicate the segment of the neutral line in the corona where the magnetic polarity change matches that of sunspots in a given hemisphere (from Loumou et al. 2018)

regions, such as area or field strength, and also the outward expansion factor of open field regions may play a role. However, in general, the north-south asymmetry and its relation to asymmetries observed in the solar activity points to the existence of an asymmetry in the solar dynamo.

\subsubsection{The Hale Sector Boundaries and Active Longitudes}

In the seventies, a combined analysis of the interplanetary magnetic field polarity, organized in sectors of negative and positive sign - toward and away from the Sun -, typically separated by two or four sector boundaries, and the emission of the Fe XIV green corona, observed during cycles $18-20$ in a wide latitude band from $57.5^{\circ} \mathrm{N}$ to $57.5^{\circ} \mathrm{S}$, showed the existence of a correlation between the interplanetary polarity and coronal patterns. Two effects were singled out: the existence of a well-defined organization of the coronal emission relative to the heliospheric magnetic polarity pattern and a reversal of the coronal pattern from one solar cycle to the next one, clearly associated with the change in leading and following polarity of sunspot groups in each solar hemisphere (Antonucci 1974b; Antonucci and Duvall 1974). That is, in a given hemisphere, the coronal emission was found to be significantly enhanced in the vicinity of the projections, back to the solar disk, of the interplanetary sector boundary separating polarities matching the leading and following sunspot polarities, for this reason named Hale sector boundary (Svalgaard and Wilcox 1976). Figure 11 (from Loumou et al. 2018) illustrates this effect in odd and even solar cycles.

The concept of interplanetary sector boundary has evolved to indicate the intersection of the warped surface of the heliospheric current sheet with the Earth, or in general a spacecraft, orbital plane. Schulz (1973) interpreted the quasi-stationary sector structure corotating with the Sun observed by Wilcox and Ness (1965), as a result of the fact that the solar wind extends the heliomagnetic equator into one warped annular surface, which intersecting the ecliptic gives origin to the polarity sectors. Hence, a simple tilted dipole originates a twosector structure, during the weakest phases of the cycle, whereas, a four-sector structure is present when a quadrupolar component, relatively stronger, associated with the dipole induces in the heliospheric current sheet a warp and tilt relative to the solar rotation axis (Getachew et al. 2017; Loumou et al. 2018, and references therein). 
The green corona is naturally organized within the heliospheric and coronal magnetic field polarity pattern in such a way that the emission is enhanced in the vicinity of the Hale portion of the neutral line of the large-scale coronal magnetic field. The regions where the neutral line separates magnetic polarities matching that of the sunspot polarities in a given hemisphere are sites not only of intensified coronal emission but also the preferred longitudes for the occurrence of associated solar phenomena such as, for instance, the formation of sunspots (Akasofu 2015) and the emergence of enhanced photospheric magnetic field, studied by Getachew et al. (2017). This suggests that the emergence of magnetic flux and sunspots at preferred longitudes warps the heliomagnetic equator, thus giving origin to the Hale boundaries.

Getachew et al. (2017) note that in cycles 21-24 the northern hemisphere displayed a more organized Hale pattern than the southern one, especially in the maximum and declining phases of the solar cycle. Furthermore, in each hemisphere a strengthening of X-ray flare activity, detected with the RHESSI space observatory (Lin et al. 2002), occurs at times when the Hale boundary is expected to be at central meridian on the solar disk (Svalgaard et al. 2011; Hudson et al. 2014). Taking into account almost two solar cycles, 23-24, of data, Loumou et al. (2018) finds that the association of flare concentration close to the Hale boundaries exists for the full spectrum of flares, from large X-class flares down to microflares, although the association between flare activity and Hale boundary during cycle 24 results to be weaker especially in the northern hemisphere.

Of course, the characteristic pattern of coronal emission, magnetic field emergence and flaring activity associated with the Hale boundaries implies the presence of preferred longitudes of electromagnetic emission or magnetic field emergence close to the Hale boundaries, as well as a longitudinal shift of the northern regions of enhanced emission/emergence relative to the southern ones, due to the relative displacement of the Hale boundaries in the two hemispheres. The extent of the longitudinal shift is determined by the presence of a two or four sector structure. The occurrence of preferred or active longitudes and hemispheric asymmetries of such longitudes is well known and was found by studying the distribution of various solar features. As an example, the sunspot data collected over the past 120 years show in each hemisphere the existence of two persistent active longitudes, where sunspots are preferentially appearing, separated by $180^{\circ}$ (Berdyugina and Usoskin 2003; Usoskin et al. 2005; Zhang et al. 2011; Mandal et al. 2017). Sunspots are preferably concentrated in one of the active longitudes which is dominant at a given time and there is a periodic alternation of active longitudes. The alternation cycle is different in the north and south hemisphere. This complex behavior is suggested to be due to the interplay of rigid and differential rotation.

Li (2011) finds that long-lived large-scale coronal streamers observed in extreme ultraviolet with the EIT instrument on SOHO are associated with the longitudes where sunspots are preferentially formed. Recurrent coronal holes also have a tendency to appear at preferred longitudes on the solar disk (Prabhu et al. 2018). Flares in the cycles 19-23 tend to cluster at preferred active longitudes, as already mentioned, and the distribution of flares is different in the two hemispheres (Shelke and Verna 1985; Bai 2003; Joshi et al. 2006). In addition, the same behavior is ascribed to those active regions which show a high capability of producing coronal mass ejections (Gyenge et al. 2017).

\subsection{Coronal Rotation}

Notwithstanding the large-scale, long-lived magnetic fields contribute less than $10 \%$ of the total solar magnetic flux, they are crucial not only in shaping the global corona and guiding 
the expansion of the solar wind, as discussed in the preceding sections, but also in regulating the rotation of the solar corona as well as the polarity pattern and rotation of the magnetic field in the heliosphere. Magnetic features with shorter life-times and smaller spatial scales are known to be characterized by differential rotation, namely, their rotation rate is varying with latitude (e.g., Howard and Harvey 1970). On the other hand, large-scale long-lived field patterns, primarily arising from the dipolar component and lower order multipoles of the solar magnetic field, are observed to rotate less differentially and differently in the two hemispheres and manifest a clear asymmetry with respect to the equator for periods of time of the order of the solar cycle. The fact that tracers of the large-scale photospheric magnetic field are organized according to a persistent pattern with different rotation in the two hemispheres of the Sun points to a relatively weak coupling between magnetic fields as they emerge in the opposite hemispheres. This behavior of the large-scale long-lived magnetic field patterns is much different than that of the total magnetic flux that instead exhibits rotational properties nearly symmetric around the equator.

\subsubsection{Magnetic Field Rotation}

A Fourier analysis of a 10 years long time series of the photospheric magnetic field detected at the Wilcox Solar Observatory, WSO, covering most of cycle 21 from 1976 to 1986, performed in order to determine the rotation rate as a function of latitude of the long-lived large-scale features, shows that the field emerges from the photosphere in a coherent and persistent pattern consisting of two quite broad latitude belts that rotate rigidly. A 26.9 day synodic period is dominant in a zone $24^{\circ}$ wide centered at $15^{\circ}$ in the northern hemisphere, and a 28.1 day period predominates in the southern hemisphere, in a zone $32^{\circ}$ wide centered near $26^{\circ}$ (Antonucci et al. 1990). In addition, the northern sources of the photospheric field are organized according to a four-sector configuration, while the southern source is probably organized in two sectors. When, on the other hand, the magnetic field rotation is studied over intervals much shorter than the solar cycle, the region of enhanced power present in the southern hemisphere shows a drift in latitude and frequency along the sunspot differential rotation curve in agreement with the migration in latitude of sunspot activity. On the contrary, the rotation pattern present in the northern hemisphere retains approximately the same dominant latitude-frequency values, except during the late declining phase. This is evidence for a significant contribution of individual long-lived active regions to the rotation frequency in the southern hemisphere, not detectable when the analysis is performed on a solar cycle time-scale, which is either absent or reduced in the northern hemisphere.

The rotational properties of the solar photospheric magnetic field with asymmetric behavior with respect to the equator might be a characteristic persisting at least since cycle 19. Indeed, the same pattern of a northern zone rotating rigidly with a synodic period of 27.0 days and a southern zone - consisting of shorter-lived features - rotating at 28.1 days, partially overlapping in latitude the zones identified in the study of the WSO data, can be inferred from the plots of a study of the photospheric magnetic field observed at Mount Wilson Observatory, in the years 1959-1967 during cycle 19 (Wilcox et al. 1970).

Coronal Magnetic Field Rotation Approximately the same frequencies dominant in the long-lived pattern of the photospheric magnetic field over cycle 21 are present also in the rotation of the extrapolated coronal magnetic fields at the source surface, set at $2.5 R_{\odot}$, where the field lines are assumed to be radially stretched outward by the solar wind. However, at the level of the source surface, the rigidly rotating zones display a larger latitudinal extent (Hoeksema and Scherrer 1987). Badalyan and Obridko (2018a,b) extended the study 
of coronal magnetic field rotation to the years from 1976 to 2004 including three consecutive solar cycles, namely, 21, 22 and 23 . Fields were extrapolated out to $2.45 R_{\odot}$ and their rotation was analyzed as function of latitude, heliodistance and phase of solar activity. Notwithstanding a different approach in selecting the lifetime of the magnetic features contributing to the rotation signal, their analysis confirms the presence of north-south asymmetries in the rotation of the coronal field and leads to the conclusion that the gradient of differential rotation with latitude significantly varies with the phase of the solar cycle, and decreases with increasing heliocentric distance, although some degree of differential rotation is retained even close to the source surface. In addition, the equatorial rotation period gradually increases with height in the range between 1.2 to $1.9 R_{\odot}$.

It is interesting to note that in a study of rotation of the preferred longitudes for flare occurrence from cycle 19 to 23, Bai (2003) finds different rotation rates in the two hemispheres consistent with the large-scale photospheric magnetic field results. That is, the rotation rates are close to 27 days in the northern hemisphere, in cycles 19-21, and 28 days in the southern hemisphere in cycles 19-21 and 23. Addressing the problem of the magnetic field rotation by considering the Sun as a star, Mordvinov and Plyusnina (2000) find that in the years 1968-2000 during the phase of rising activity the dominant rotational modes of the solar mean magnetic field have periods of 27.8-28.0 days, whereas the 27-day rotational mode dominates the phase of decreasing activity.

At all phases of the cycle except times of polar field reversals, the large-scale, low-order fields, particularly the axisymmetric dipole and octupole, generally dominate the global coronal morphology (e.g., Petrie 2013). In addition, at higher heliocentric distance the structure and rotation rate of the corona is determined by increasingly large-scale magnetic patterns related to the lower order harmonics of the solar magnetic field, whilst the contribution of the higher harmonics has become negligible. Thus, in absence of coronal transients, the solar global field contributes in a significant manner to structure the heliospheric magnetic field. That is, the tendency of large-scale, long-lived magnetic fields to rotate as a rigid body in quite broad latitude belts and the north-south asymmetry in rotation determine the properties of the interplanetary magnetic field and its polarity pattern. The most significant coronal field periodicities with lifetime comparable to a solar cycle, namely, the 26.9 and 28.1 day periodicities - the slower rotation being detected in the southern hemisphere - are observed over a significant range of latitudes, thus, they are retained when converting the source surface magnetic field intensity values in polarity values, with the northern magnetic field organized according to a four sector configuration and the southern field in two sectors, consistently with the behavior of the photospheric magnetic field (Antonucci et al. 1990).

Interplanetary Magnetic Field Rotation The dominant periodicities in the interplanetary magnetic field polarity, namely, 27 day and 28.5 day characteristic of different phases of the solar cycle, persisting for at least six solar cycles (Svalgaard and Wilcox 1975), are approximately the same that have been derived for the long-lived, large-scale photospheric and coronal fields in the opposite hemispheres, 26.9 and 28.1 day. This analogy would suggest that the four-sector polarity structure of the interplanetary magnetic field is primarily related to the northern hemisphere and the two-sector structure to the opposite hemisphere.

According to Wang (2014), the north-south asymmetries in the distribution of active regions, reflecting those in the underlying distribution of the photospheric magnetic flux, give rise to hemispheric asymmetries in the coronal rotation rate. During the late declining phase of the cycle, the coronal field which tends to reduce to a dipole rotates nearly rigidly with a period close to 27 days. During the rising phase of the cycle, on the other hand, nonaxisymmetric photospheric flux concentrated in the mid-latitude active regions, is leading 
to a slower, less rigid rotation of the coronal field. The slower rotation characteristic of the rising phase is also evident in the $\sim 28$-day recurrence rate of the interplanetary magnetic field sector structure, which then switches to a 27-day pattern during the declining phase. The coexistence of different rotation rates in the two hemispheres may also suggest a preferential link of the heliosphere, measured at the ecliptic plane, to one hemisphere or to the other during different parts of the cycle.

\subsubsection{Rotation of the Large-Scale Corona}

The strict dependence of coronal rotation on the magnetic field properties is confirmed by several studies on the electron and ion components of the corona. However, the scenario emerging from the various analyses is not quite homogeneous due to differences in diagnostic techniques and in the kind of tracers assumed to determine coronal rotation. In any case, as a general rule the degree of differential rotation of the corona with latitude is a function of the solar cycle and tends to decrease in the declining part of the cycle. Moreover, the rotation of coronal structures does reflect the tendency of the large-scale magnetic field patterns to rotate rigidly in quite broad latitude belts.

Rotation as a Function of Heliolatitude Since tracers cannot be easily identified in the limb observations of the white light scattered by the electron corona, the rotational properties are deduced by analyzing the periodicities existing in the modulation of the coronal emission which is due to the presence of recurrent patterns having lifetime of a rotation period or more. During the ascending phase of cycle 20, in the interval 1964-1967, Hansen et al. (1969) found persistent coronal features reappearing at the limb for several rotations that at mid latitudes rotate at a faster rate than the underlying photosphere, with a degree of differential rotation varying from year to year and from one hemisphere to the other one. The analyses of a more extended interval of data of the white light K-corona from 1964 to 1983, combining the studies of Parker et al. (1982) and Fisher and Sime (1984), confirm that coronal tracers, within $\pm 60^{\circ}$ in latitude, tend to rotate more rapidly than photospheric tracers, such as sunspots, and much less differentially, except at the equator where the coronal rotation rate is close to the sunspot rate.

Rotational properties are variable through the solar cycle and this variability clearly points to a coexistence of rigid rotation of long-lived features and differentially rotating shorter-lived coronal features, dominant in the rising and maximum phase of solar activity, as in the case of the magnetic fields. In the years of declining solar activity of three consecutive solar cycles, namely 18, 19 and 20 spanning the interval 1947-1974, the green corona detected at the limb in the Fe XIV line $(530.3 \mathrm{~nm})$ was observed to rotate in a nearly rigid manner in a wide latitude range, $\pm 57.5^{\circ}$, with a synodic period close to 27 days (Antonucci and Svalgaard 1974; Antonucci and Dodero 1977). However, in this phase of the solar cycle, coronal features persisting more than one synodic rotation period, affected by little or no differential rotation, coexist with shorter lived features differentially rotating (Antonucci and Dodero 1979). Hence, rigid and differential rotation coexist even in the declining phase of a cycle. The coexistence of rigid and differential rotation reflects the difference in behavior of individual magnetic features affected by differential rotation and the large-scale magnetic pattern rigidly rotating. Wang and Sheeley (1994) propose to interpret the rigidly rotating pattern as due to transport of magnetic flux across latitude, that sets up a stationary pattern in the 27-day frame, in alternative to the hypothesis of continual emergence of magnetic flux highly organized on a global scale.

Further analyses of the green corona by Altrock (2003), relative to the two successive solar cycles, 21 and 22 from 1976 to 2001, confirm that in approximately the same latitude 
range, up to $60^{\circ}$, the rotation measured at 1.15 and $1.25 R_{\odot}$ varies from essentially rigid near solar minimum to differential in the rising phase of the cycle. At latitudes above $60^{\circ}$, however, the rotation is almost exactly out of phase with respect to the lower latitude variation, being nearly rigid in the rising phase of the cycle and strongly differential near solar minimum (Altrock 2003).

During solar minimum, coronal streamers are limited to low latitudes and share the rotation properties of these zones. On the other hand, when streamers form at high latitudes, they exhibit considerable differential rotation, as shown by Morgan and Habbal (2010) by applying solar rotational tomography to the total brightness of the corona observed at $4 R_{\odot}$ with the LASCO coronagraph C2. Morgan (2011) identifies not only the rotational signatures of long-lived patterns, substantially consistent with those derived from the studies of emission modulation, but also detects the effects of rapid structural reconfigurations occurring in the outer corona.

Coronal Hole Rotation When the first observations of the corona against the disk in EUV, in the Fe XV line, and in X-ray emission, obtained with space instruments on the OSO7 and Skylab missions, allowed the identification of coronal holes as features linked to regions of unipolar magnetic field with dimmed emission, a large hole persisting from May 1972 to October 1973 during cycle 20 was studied by Wagner (1975) and Timothy et al. (1975). The hole was rotating rigidly at a rate consistent with the rate derived for the green corona (Antonucci and Svalgaard 1974), confirming that rigid rotation is a characteristics associated with large-scale long-lived coronal patterns.

Recently, extending previous analyses (e.g., Insley et al. 1995) to the period 1992-2016 from cycle 22 to 24, Prabhu et al. (2018) studied the recurrent coronal holes on the basis of an extensive body of observations from ground based (He I $1083.0 \mathrm{~nm}$ ) and space instrumentation (SOHO EIT and STEREO Secchi EUVI, SDO AIA $19.5 \mathrm{~nm}$ and $19.3 \mathrm{~nm}$ images). The number of recurrent coronal holes varies from cycle to cycle and a latitudinal asymmetry, reversing from cycle 23 to cycle 24, is observed in the number of recurrent coronal holes, that also have a tendency to appear at preferred longitudes on the solar disk (Prabhu et al. 2018). The values of the equatorial rotation rate determined in this analysis, $13.23 \mathrm{deg} / \mathrm{day}$ with a latitude gradient $-0.45 \mathrm{deg} / \mathrm{day}$, confirm the results from previous analyses by Insley et al. (1995), Japaridze et al. (2015) and those obtained from green line measurements by Antonucci and Dodero (1977). These findings are a further evidence that coronal holes are either intrinsic elements of the global coronal structure organized by the large-scale magnetic fields that does not share the properties of differential rotation, or, alternatively, patterns of open field lines that are not permanently tied to the differentially rotating component of the photospheric plasma. The fact that the rotation curve of coronal holes matches the rotation of the interior of the Sun at $0.5 R_{\odot}$, as inferred from helioseismology, leads Prabhu et al. (2018) to suggest that coronal holes are rooted below the convection zone, (see also Bagashvili et al. 2017). The scenario is different when considering mid-latitude coronal holes present during solar maximum years, those rotation deviates from rigid rotation, but they still rotate more rigidly than sunspots (Insley et al. 1995; Oghrapishvili et al. 2018).

It is interesting to note that a study of coronal rotation of the Sun as a star, based on the daily $10.7 \mathrm{~cm}$ solar radio emission flux during a period covering almost 7 solar cycles, shows that the rotation periods display a significant decreasing trend. That is, globally the corona tends to rotate faster with time (Xie et al. 2017). This may well be related to a decreasing contribution to the radio emission of coronal features rotating more slowly associated with solar activity which is indeed decreasing throughout this time interval. This interpretation would be consistent with an observed decrease in solar rotation with increasing solar activity (Obridko and Shelting 2016). Parker et al. (1982) also notes that the lower corona 
at $1.125 R_{\odot}$ during cycle 20 rotated faster than did recurrent sunspots during cycles 12 through 17 .

Coronal Rotation as a Function of Heliodistance The results of the studies of coronal rotation as a function of altitude above the limb are not quite homogeneous. However, in general, the corona rotates more rapidly with increasing height in an inner narrow layer, then substantially rigidly in the intermediate zone up to $2-2.5 R_{\odot}$, and slows down above $2.5 R_{\odot}$. The rotation of the inner layer of the corona between 0.08 and $1.18 R_{\odot}$ was determined by analyzing the disk-integrated radio flux (Vats et al. 2001). From 1.125 to $1.5 R_{\odot}$, the coronal rotation variation with height depends on the latitude zone (Parker et al. 1982): an increase in rotation rate is observed at latitudes $40^{\circ}-60^{\circ}$, while the opposite behavior is found at low and mid latitudes. On the other hand, in a partially overlapping latitude layer, from 1.125 to $2 R_{\odot}$, a nearly rigid rotation with height of the electron corona was observed by Hansen et al. (1969). In addition, Newkirk (1967) reports a study of a coronal streamer rotating rigidly with height between 2.2 and $2.6 R_{\odot}$.

The SOHO coronagraphs extended coronal observations further out. At the end of cycle 22, from May 1996 to May 1997, the LASCO coronagraphs detected a persistent stable asymmetry in brightness, both of the Fe XIV emission and the electron scattered light, affected by almost rigidly rotational properties. The emission modulation allowed Lewis et al. (1999) to determine coronal rotation out to $15 R_{\odot}$, obtaining the following results: the observed green corona rotates in 27.4 days below $2 R_{\odot}$, whereas, a slower rotation rate, corresponding to a 27.7 day period, is measured on the basis of the white light observations above $2.5 R_{\odot}$ (Lewis et al. 1999). A similar behavior is deduced from a study of the $\mathrm{O}$ VI $(103.2 \mathrm{~nm})$ and H I Lyman- $\alpha(121.6 \mathrm{~nm}) \mathrm{UV}$ line emission observed with UVCS in the solar minimum phase; the equatorial region of the limb corona is rotating almost rigidly with altitude from $1.5 R_{\odot}$ out to about $2.3 R_{\odot}$, beyond this level the rotation rate decreases (Giordano and Mancuso 2008). The UVCS data analyzed throughout solar cycle 23, however, show that the equatorial coronal rotation is almost rigid out to $3 R_{\odot}$, except in three limited intervals (two intervals at solar minimum and one at maximum of activity) when beyond the source surface level the rotation rate varies abruptly, either increasing or decreasing with respect to the inner zone (Mancuso and Giordano 2012).

In summary, coronal observations show that the large-scale corona in general rotates more rigidly than the underlying photosphere and the rotation tends to become increasingly rigid with height up to at about $2.5 R_{\odot}$, region where the plasma is controlled by the magnetic field. This behavior can be ascribed to the rapid decrease of higher order multipoles of the magnetic field above the photosphere. Hence, the dipolar configuration of the coronal magnetic field become increasingly dominant, and depending on the phase of the solar cycle, the rotation becomes more and more rigid. The dependence of the latitudinal profile of the rotation rate on the solar cycle is then related to the latitudinal distribution of the large-scale, non-axisymmetric photospheric flux associated with the emergence and decay of active regions (Wang 2014).

North-South Asymmetry in Coronal Rotation Whilst latitudinal asymmetries in rotation have been not always observed for photospheric tracers or in the velocity signal measured at photospheric level, it is evident that significant north-south asymmetries are clearly present in the rotation rate of the long-lived photospheric and coronal magnetic fields (e.g. Hoeksema and Scherrer 1987; Antonucci et al. 1990), as well as in the coronal emission (e.g., Hansen et al. 1969; Prabhu et al. 2018), being coronal rotation controlled by the magnetic field. Indeed, for instance, the slower rotation rate of the large-scale magnetic fields 
observed in the southern hemisphere is also present in the UV corona (Giordano and Mancuso 2008). The existence of a persistent hemispheric difference in rotation is confirmed by the study of the radio images at $17 \mathrm{GHz}$ of $\mathrm{H} \alpha$ filaments, in cycles 19-22 (Vats et al. 2001; Gigolashvili et al. 2005, 2011), and of the sunspot rotational behavior over the interval 1874-2009 (Zhang et al. 2011), who find that the mean rotation in the southern hemisphere is slower than in the northern one. Furthermore, a systematic long-term north-south asymmetry of the sunspot rotation has been found, which oscillates with about a 80-90 year period. The hemispheric anticorrelation peaks around 1920 (fastest rotation in the southern hemisphere) and 1980 (fastest rotation in the northern hemisphere) and goes through a minimum in cycles 18-20 (Zhang et al. 2013). These results point to the existence of a weak coupling of the magnetic field rotation in the opposite hemispheres, that is, a weak interdependence of the field systems originating in the two hemispheres. On the other hand, the long-term oscillation in the relative rotation rates of the two hemispheres suggests that there is a systematic interchange of angular momentum between the two hemispheres (Zhang et al. 2013).

A plausible interpretation of the north-south asymmetry in the rotation of the photospheric fields and consequently of the corona, would be that the magnetic patterns originate at different depths in the solar convection zone. In this hypothesis, for instance, the variation of the dominant periodicity of the magnetic fields in the southern hemisphere during cycle 21 (Antonucci et al. 1990) would be ascribed to a variation in depth of the level of the roots of the magnetic fields. Similar conclusions can be inferred from coronal hole studies. Gilman (1977) proposes that the rotation of coronal holes can be ascribed to their deep rooting in the convection zone. When studying coronal holes independently of their lifetime, Bagashvili et al. (2017) infer that in the years 2013-2015 these magnetic features are linked to the tachocline zone and lower layers of the convection zone. On the same line, Prabhu et al. (2018) suggest an anchoring of recurrent coronal holes to layers below the convection zone. Their conclusions are derived from a comparison of the rotation of the emergent magnetic field and associated coronal pattern, with the rotation rate as a function of depth in sub-photospheric layers as inferred with helioseismology techniques (e.g., Schou et al. 1998; Kosovichev et al. 1997; Thompson et al. 2003; Antia and Basu 2010). Further in-depth studies are indeed needed to confirm the link of coronal tracers to their sub-surface source and contribute to explain the north-south asymmetries found in the rotational properties of the solar atmosphere.

\subsection{Future Observations of the Large-Scale Corona}

The phenomenological picture emerging from decades of study of coronal holes highlights their fundamental importance due to their nature of open field regions in the formation of the heliosphere, its structure and dynamics, and confirms the link of these dim large-scale coronal features with the magnetic cycles of the Sun and, in turn, with the not yet fully understood dynamo action and its asymmetries and anomalies.

The Solar Orbiter telescopes will explore for the first time the magnetic and velocity fields in the polar regions, this allowing to directly investigate the source region of the fast wind in the core of polar coronal holes. In addition, the subsurface layers of the source of the fast wind will be probed by studies of the local helioseismology. At present the sub-surface structure around the poles is very poorly known. Observations of the magnetic field and the sound speed below the surface of the polar region will provide new insights into the working of the solar dynamo, which is responsible for enhancing the magnetic field that forms the roots of the large-scale corona. 
The out-of-ecliptic observations of the corona will allow to assess the true longitudinal distribution of streamers and determine their lifetime during the different phases of the solar cycle. These results cannot be obtained with the present remote sensing instrumentation due to the fact that the observations of the outer corona are limited to their passage at the limb where the coronal features are visible for a short time interval due to solar rotation.

At least during solar minimum when the coronal configuration is relatively simple, the observational description of the solar wind is fairly well outlined, although it needs to be further consolidated. When the level of solar activity becomes significant, on the other hand, the identification of the solar wind sources requires still a substantial observational effort to investigate the more complex coronal structure and dynamics, and the conditions of the wind close to the Sun in order to establish a unambiguous link of the heliospheric wind with its solar source.

The deficiencies of the present knowledge highlight the importance of complementary observations of the fast and slow wind streams at coronal and inner-heliospheric level. The dynamics of wind streams can indeed be fully understood when complementing the results obtained by applying coronagraphic diagnostic techniques - such as Doppler dimming - in order to derive global maps of the outflows in the corona, as foreseen with the Solar Orbiter remote sensing instrumentation, with in situ measurements of the physical parameters of the wind in the inner heliosphere, as performed with Parker Solar Probe and the Solar Orbiter in situ instruments observing the Sun from a vantage point out of the ecliptic plane. This approach will allow us to derive the physical characteristics of the wind streams all along their path during the propagation from the corona outward and avoid any ambiguity when mapping wind streams back from interplanetary space to the Sun.

Funding Note Open access funding provided by Istituto Nazionale di Astrofisica within the CRUI-CARE Agreement.

Publisher's Note Springer Nature remains neutral with regard to jurisdictional claims in published maps and institutional affiliations.

Open Access This article is licensed under a Creative Commons Attribution 4.0 International License, which permits use, sharing, adaptation, distribution and reproduction in any medium or format, as long as you give appropriate credit to the original author(s) and the source, provide a link to the Creative Commons licence, and indicate if changes were made. The images or other third party material in this article are included in the article's Creative Commons licence, unless indicated otherwise in a credit line to the material. If material is not included in the article's Creative Commons licence and your intended use is not permitted by statutory regulation or exceeds the permitted use, you will need to obtain permission directly from the copyright holder. To view a copy of this licence, visit http://creativecommons.org/licenses/by/4.0/.

\section{References}

L. Abbo, E. Antonucci, Z. Mikić et al., Adv. Space Res. 46, 1400 (2010)

L. Abbo, R. Lionello, P. Riley, Y.-M. Wang, Sol. Phys. 290, 2043 (2015)

L. Abbo, L. Ofman, S.K. Antiochos et al., Space Sci. Rev. 201, 55 (2016)

L. Abbo, S. Giordano, L. Ofman, Astron. Astrophys. 623, A95 (2019)

S.-I. Akasofu, Geophys. Res. Lett. 42, 2571 (2015)

H. Alfvén, Mon. Not. R. Astron. Soc. 107, 211 (1947)

L.A. Allen, S.R. Habbal, Y.Q. Hu, J. Geophys. Res. 103, 6551 (1998)

R.C. Altrock, Sol. Phys. 213, 23 (2003)

H.M. Antia, S. Basu, Astrophys. J. 720, 494 (2010)

S.K. Antiochos, J.A. Linker, R. Lionello et al., Space Sci. Rev. 172, 169 (2012)

E. Antonucci, Eldo-Cecles/Esro-Cers Sci. Tech. Rev. 6, 17 (1974a)

E. Antonucci, Sol. Phys. 34, 471 (1974b) 
E. Antonucci, Plasma dynamics and diagnostics in the solar transition region and corona, in ESA, vol. SP-446 (1999), p. 53

E. Antonucci, Space Sci. Rev. 124, 35 (2006)

E. Antonucci, M.A. Dodero, Sol. Phys. 53, 179 (1977)

E. Antonucci, M.A. Dodero, Sol. Phys. 62, 107 (1979)

E. Antonucci, T.L. Duvall, Sol. Phys. 38, 439 (1974)

E. Antonucci, S. Giordano, Joint SOHO/ACE workshop solar and galactic composition, in AIP Conf. Proc., vol. 598, ed. by R.F. Wimmer-Schweingruber (AIP, Melville, 2001), p. 77

E. Antonucci, L. Svalgaard, Sol. Phys. 34, 3 (1974)

E. Antonucci, J.T. Hoeksema, P.H. Scherrer, Astrophys. J. 360, 296 (1990)

E. Antonucci, S. Giordano, C. Benna, J.L. Kohl, G. Noci, J. Michels, S. Fineschi, The corona and solar wind near minimum activity, in Proc. Fifth SOHO Workshop, ed. by A. Wilson. ESA, vol. SP-404, (ESA, Noordwijk, 1997), p. 175

E. Antonucci, M.A. Dodero, S. Giordano, Sol. Phys. 197, 115 (2000)

E. Antonucci, L. Abbo, M. Dodero, Astron. Astrophys. 435, 699 (2005)

E. Antonucci, L. Abbo, D. Telloni, Space Sci. Rev. 172, 5 (2012)

M.J. Aschwanden, H. Peter, Astrophys. J. 840, 4 (2017)

R.G. Athay, O.R. White, Astrophys. J. 226, 1135 (1978)

R.G. Athay, O.R. White, Astrophys. J. 39, 333 (1979)

O.G. Badalyan, V.N. Obridko, Sol. Phys. 293, 128 (2018a)

O.G. Badalyan, V.N. Obridko, Astron. Lett. 44, 727 (2018b)

S.R. Bagashvili, B.M. Shergelashvili, D.R. Japaridze et al., Astron. Astrophys. 603, A134 (2017)

T. Bai, Astrophys. J. 585, 1114 (2003)

D. Baker, L. van Driel-Gesztelyi, C.H. Mandrini, P. Démoulin, M.J. Murray, Astrophys. J. 705, 926 (2009)

A. Bemporad, W.H. Matthaeus, G. Poletto, Astrophys. J. Lett. 677, L137 (2008)

S.V. Berdyugina, I.G. Usoskin, Astron. Astrophys. 405, 1121 (2003)

I.A. Bilenko, Sol. Phys. 293, 106 (2018)

R.L. Blake, T.A. Chubb, H. Friedman, A.E. Unzicker, Astrophys. J. 137, 3 (1963)

J.D. Bohlin, Sol. Phys. 51, 377 (1977)

J.D. Bohlin, N.R. Sheeley Jr., Sol. Phys. 56, 125 (1978)

S. Bravo, G.A. Stewart, Astrophys. J. 489, 992 (1997)

D.H. Brooks, H.P. Warren, I. Ugarte-Urra, A.R. Winebarger, Astrophys. J. Lett. 772, L19 (2013)

D.H. Brooks, I. Ugarte-Urra, H.P. Warren, Nat. Commun. 6, 5947 (2015)

G.E. Brueckner, R.A. Howard, M.J. Koomen et al., Sol. Phys. 162, 357 (1995)

R. Bruno, D. Telloni, L. Sorriso-Valvo, Astron. Astrophys. 627, A96 (2019)

Y. Chen, R. Esser, L. Strachan et al., Astrophys. J. 602, 415 (2004)

I.-H. Cho, Y.-J. Moon, V.M. Nakariov et al., Phys. Rev. Lett. 121(7), 075101 (2018)

J.W. Cirtain, L. Golub, A.R. Winebarger et al., Nature 493, 501 (2013)

O. Cohen, Sol. Phys. 290, 2245 (2015)

S.R. Cranmer, L.K. Kohl, G. Noci et al., Astrophys. J. 511, 481 (1999a)

S.R. Cranmer, G.B. Field, J.L. Kohl, Astrophys. J. 518, 937 (1999b)

S.R. Cranmer, S.E. Gibson, P. Riley, Space Sci. Rev. 212, 1345 (2017)

J.L. Culhane, E. Hiei, G.A. Doschek et al., Sol. Phys. 136, 89 (1991)

J.L. Culhane, L.K. Harra, A.M. James et al., Sol. Phys. 243, 19 (2007)

I. De Moortel, P. Browning, Philos. Trans. R. Soc. Lond. Ser. A 373, 20140269 (2015)

B. De Pontieu, S.W. McIntosh, M. Carlsson et al., Science 318, 1574 (2007)

G. de Toma, Sol. Phys. 274, 195 (2011)

G. Del Zanna, B.J.I. Bromage, J. Geophys. Res. 104, 9753 (1999)

G. Del Zanna, H.E. Mason, Astron. Astrophys. 406, 1089 (2003)

J.-P. Delaboudiniere, J.E. Artzner, J. Brunaud et al., Sol. Phys. 162, 291 (1995)

L.H. Deng, Y.Y. Xiang, Z.N. Qu, J.M. An, Astrophys. J. 151, 70 (2016)

D. Dobrzycka, S.R. Cranmer, A.V. Panasyuk et al., J. Geophys. Res. 104, 9791 (1999)

S. Dolei, R. Susino, C. Sasso et al., Astron. Astrophys. 612, A84 (2018)

V. Domingo, B. Fleck, A.I. Poland, Sol. Phys. 162, 1 (1995)

G.A. Doschek, Sol. Phys. 86, 9 (1983)

S.J. Edwards, C.E. Parnell, L.K. Harra, J.L. Culhane, D.H. Brooks, Sol. Phys. 291, 117 (2016)

R. Fisher, D.G. Sime, Astrophys. J. 287, 959 (1984)

H. Friedman, Sun and Earth (Scientific American Library, New York, 1986)

J. Geiss, P. Hirt, H. Leutwyler, Sol. Phys. 12, 458 (1970)

T. Getachew, I. Virtanen, K. Mursula, Sol. Phys. 292, 174 (2017)

S.E. Gibson, A. Fludra, F. Bagenal et al., J. Geophys. Res. 104, 9691 (1999a) 
S.E. Gibson, D. Biesecker, M. Guhathakurta et al., Astrophys. J. 520, 871 (1999b)

M.S. Gigolashvili, D.R. Japaridze, T.G. Mdznarishvili et al., Sol. Phys. 227, 27 (2005)

M.S. Gigolashvili, D.R. Japaridze, T.G. Mdznarishvili, Astrophysics 54, 653 (2011)

P.A. Gilman, Astron. Astrophys. 58, 315 (1977)

S. Giordano, S. Mancuso, Astrophys. J. 688, 656 (2008)

L. Glesener, S. Krucker, S. Christe et al., in SPIE Proc., vol. 9905E (2016)

G. Gloeckler et al., Astron. Astrophys. Suppl. Ser. 92, 267 (1992)

N. Grevesse, A.J. Sauval, Space Sci. Rev. 85, 161 (1998)

N. Gyenge, T. Singh, T.S. Kiss et al., Astrophys. J. 838, 18 (2017)

S.R. Habbal, R. Woo, S. Fineschi, R. O’Neal, J. Kohl, G. Noci, C. Korendyke, Astrophys. J. Lett. 489, L103 (1997)

S.R. Habbal, R. Woo, J.-C. Vial, Space Sci. Rev. 97, 5 (2001)

B.N. Handy, L.W. Acton, C.C. Kankelborg et al., Sol. Phys. 187, 229 (1999)

R.T. Hansen, S.F. Hansen, H.G. Loomis, Sol. Phys. 10, 135 (1969)

L.K. Harra, T. Sakao, C.H. Mandrini et al., Astrophys. J. 676, 147 (2008)

R.A. Harrison, E.C. Sawyer, M.K. Carter et al., Sol. Phys. 162, 233 (1995)

K.L. Harvey, F. Recely, Sol. Phys. 211, 31 (2002)

T. Hiltula, K. Mursula, Geophys. Res. Lett. 33, L03105 (2006)

J.T. Hoeksema, P.H. Scherrer, Astrophys. J. 318, 428 (1987)

S.J. Hofmeister, A. Veronig, M.A. Reiss, M. Temmer, S. Vennerstrom, B. Vrsnǎk, B. Heber, Astrophys. J. 835, 268 (2017)

T.E. Holzer, E. Leer, The corona and solar wind near minimum activity, in ESA, vol. SP-404 (1997), p. 65

R. Howard, J. Harvey, Sol. Phys. 12, 23 (1970)

R.A. Howard, J.D. Moses, A. Vourlidas et al., Space Sci. Rev. 136, 67 (2008)

H.S. Hudson, L. Svalgaard, I.G. Hannah, Space Sci. Rev. 186, 17 (2014)

A.J. Hundhausen, Coronal Expansion and Solar Wind (Springer, Berlin, 1972)

J.E. Insley, V. Moore, R.A. Harrison, Sol. Phys. 160, 1 (1995)

D.R. Japaridze, S.R. Bagashvili, B.M. Shergelasvili et al., Astrophysics 58, 609 (2015)

B. Joshi, P. Pant, P.K. Manoharan, J. Astrophys. Astron. 27, 151 (2006)

M.L. Kaiser, T.A. Kucera, J.M. Davila et al., Space Sci. Rev. 136, 5 (2008)

J.C. Kasper et al., Astrophys. J. 745, 162 (2012)

M.S. Kirk, W.D. Pesnell, C.A. Young, S.A. Hess Webber, Sol. Phys. 257, 99 (2009)

Y.-K. Ko, D.A. Roberts, S.T. Lepreti, Astrophys. J. 864, 139 (2018)

J.L. Kohl, R. Esser, L.D. Gardner et al., Sol. Phys. 162, 313 (1995)

J.L. Kohl, G. Noci, E. Antonucci et al., Sol. Phys. 175, 613 (1997)

J. Kohl, G. Noci, E. Antonucci et al., Astrophys. J. 1, 127 (1998)

R.A. Kopp, T.E. Holzer, Sol. Phys. 49, 43 (1976)

J.S. Koskela, I.I. Virtanen, K. Mursula, Astron. Astrophys. 618, A105 (2018)

A.G. Kosovichev, J. Schou, P.H. Scherrer et al., Sol. Phys. 170, 43 (1997)

S. Koutchmy, M. Livshits, Space Sci. Rev. 61, 393 (1992)

A.S. Krieger, A.F. Timothy, E.C. Roelof, Sol. Phys. 29, 505 (1973)

N. Labrosse, P. Heinzel, J.-C. Vial, T. Kucera, S. Parenti, S. Gunár, B. Schmeider, G. Kilper, Space Sci. Rev. 151, 243 (2010)

J.R. Lemen, A.M. Title, D.J. Akin et al., Sol. Phys. 275, 17 (2012)

R.H. Levine, M.D. Altschuler, J.W. Harvey, B.V. Jackson, Astrophys. J. 215, 636 (1977)

D.J. Lewis, G.M. Simnett, G.E. Brueckner, R.A. Howard, P.L. Lamy, R. Schwenn, Sol. Phys. 184, 297 (1999)

J. Li, Astrophys. J. 735, 130 (2011)

J. Li, J.C. Raymond, L.W. Acton et al., Astrophys. J. 506, 431 (1998)

R.P. Lin, B.R. Dennis, G.L. Hurford et al., Sol. Phys. 210, 3 (2002)

G.A. Linford, C.J. Wolfson, Astrophys. J. 331, 1036 (1988)

M.L. Loucif, S. Koutchmy, Astron. Astrophys. Suppl. Ser. 77, 45 (1989)

K. Loumou, I.G. Hannah, H.S. Hudson, Astron. Astrophys. 618, A9 (2018)

B.C. Low, ASP Conf. Ser. 286, 335 (2003)

C. Lowder, J. Qiu, R. Leamon, Sol. Phys. 292, 18 (2017)

D.H. Mackay, J.T. Karpen, J.L. Ballester, B. Schmeider, G. Aulanier, Space Sci. Rev. 151, 333 (2010)

R.M. MacQueen, J.A. Eddy, J.T. Gosling et al., Astrophys. J. Lett. 187, L85 (1974)

S. Mancuso, S. Giordano, Astron. Astrophys. 539, A26 (2012)

S. Mandal, S. Chatterjee, D. Banerjee, Astrophys. J. 835, 62 (2017)

D. Marocchi, E. Antonucci, S. Giordano, Ann. Geophys. 19, 135 (2001)

A.J. Marsh, D.M. Smith, L. Glesener et al., Astrophys. J. 864, 5 (2018)

P.C.H. Martens, G.H.J. Van den Oord, P. Hoyng, Sol. Phys. 96, 253 (1985) 
S.F. Martin, R. Bilimoria, P.W. Tracadas, Solar Surface Magnetism (Kluwer Academic Publishers, Dordrecht, 1994), p. 303

L. Matteini, D. Stansby, T.S. Horbury, C.H.K. Chen, Astrophys. J. Lett. 869, L32 (2018)

D.J. McComas, S.J. Bame, B.L. Barraclough et al., Geophys. Res. Lett. 25, 1 (1998)

D.J. McComas, R.W. Ebert, H.A. Elliott et al., Geophys. Res. Lett. 35, L18103 (2008)

S.W. McIntosh, R.J. Leamon, J.B. Gurman et al., Astrophys. J. 765, 146 (2013)

S.W. McIntosh, X. Wang, R.J. Leamon et al., Astrophys. J. 792, 12 (2014)

M.P. Miralles, S.R. Cranmer, A.V. Panasyuk, M. Romoli, J.L. Kohl, Astrophys. J. 549, L257 (2001)

M.P. Miralles, S.R. Cranmer, J.L. Kohl, Adv. Space Res. 33, 696 (2004)

A.V. Mordvinov, L.A. Plyusnina, Sol. Phys. 197, 1 (2000)

H. Morgan, Astrophys. J. 738, 189 (2011)

H. Morgan, S.R. Habbal, Astrophys. J. 710, 1 (2010)

H. Morgan, Y. Taroyan, Sci. Adv. 3 (2017)

J.D. Moses, E. Antonucci, F. Auchere et al., Nat. Astron. (2020). https://doi.org/10.1038/s41550-020-1156-6

R.H. Munro, B.V. Jackson, Astrophys. J. 213, 874 (1977)

R.H. Munro, G.L. Withbroe, Astrophys. J. 176, 511 (1972)

K. Mursula, T. Hiltula, Geophys. Res. Lett. 30, 2135 (2003)

V.M. Nakariakov, E. Verwichte, Living Rev. Sol. Phys. 2, 3 (2005)

W.M. Neupert, V. Pizzo, J. Geophys. Res. 79, 3701 (1974)

G. Newkirk Jr., Annu. Rev. Astron. Astrophys. 5, 213 (1967)

G. Noci, Sol. Phys. 28, 403 (1973)

G. Noci, E. Gavryuseva, Astrophys. J. Lett. 658, L63 (2007)

G. Noci, J.L. Kohl, G.L. Withbroe, Astrophys. J. 315, 706 (1987)

G. Noci et al., The corona and solar wind near minimum activity, in Proc. Fifth SOHO Workshop, ed. by A. Wilson. ESA, vol. SP-404, (ESA, Noordwijk, 1997), p. 75

J.T. Nolte, A.S. Krieger, A.F. Timothy et al., Sol. Phys. 46, 303 (1976)

V.N. Obridko, B.D. Shelting, Astron. Lett. 42, 631 (2016)

N.B. Oghrapishvili, S.R. Bagashvili, D.A. Maghradze et al., Adv. Space Res. 61, 3039 (2018)

E.L. Olsen, E. Leer, T.E. Holzer, Astrophys. J. 420, 913 (1994)

O. Panasenco, M. Velli, SOLAR WIND 13: Proceedings of the Thirteenth International Solar Wind Conference. AIP Conference Proceedings, vol. 1539 (2013), p. 50

S. Parenti, Living Rev. Sol. Phys. 11, 1 (2014)

S. Parenti, B.J.I. Bromage, G. Poletto, G. Noci, J.C. Raymond, G.E. Bromage, Astron. Astrophys. 363, 800 (2000)

S. Parenti, G. Del Zanna, J.-C. Vial, Astron. Astrophys. 625, A52 (2019)

E.N. Parker, Astrophys. J. 128, 664 (1958)

E.N. Parker, Astrophys. J. 174, 499 (1972)

G.D. Parker, R.T. Hansen, S.F. Hansen, Sol. Phys. 80, 185 (1982)

A.N. Parmar, J.L. Culhane, C.G. Rapley, E. Antonucci, A.H. Gabriel, M. Loulergue, Mon. Not. R. Astron. Soc. 197, 29 (1981)

W.D. Pesnell, B.J. Thompson, P.C. Chamberlin, Sol. Phys. 275, 3 (2012)

G.J.D. Petrie, Astrophys. J. 768, 162 (2013)

I. Plotnikov, A.P. Rouillard, J.A. Davies et al., Sol. Phys. 291, 1853 (2016)

D.I. Pontin, M. Janvier, S.K. Tiwari et al., Astrophys. J. 837, 108 (2017)

A. Posner, T.H. Zurbuchen, N.A. Schwadron et al., J. Geophys. Res. 106, 15869 (2001)

K. Prabhu, B. Ravindra, M. Hegde, V.H. Doddamani, Astrophys. Space Sci. 363, 108 (2018)

J.C. Raymond, J.L. Kohl, G. Noci et al., Sol. Phys. 175, 645 (1997)

F. Reale, Living Rev. Sol. Phys. 11, 4 (2014)

V. Réville, A.S. Brun, Astrophys. J. 850, 45 (2017)

R.L. Rosenberg, P.J. Coleman Jr., J. Geophys. Res. 74, 24 (1969)

R. Rosner, W.H. Tucker, G.S. Vaiana, Astrophys. J. 220, 643 (1978a)

R. Rosner, L. Golub, B. Coppi, G.S. Vaiana, Astrophys. J. 222, 317 (1978b)

D.M. Rust, Astrophys. J. 150, 313 (1967)

E. Sanchez-Diaz, A.P. Rouillard, J.A. Davies et al., Astrophys. J. 851, 32 (2017)

K.H. Schatten, J.M. Wilcox, N.F. Ness, Sol. Phys. 6, 442 (1969)

P.H. Scherrer, R.S. Bogart, R.I. Bush et al., Sol. Phys. 162, 129 (1995)

P.H. Scherrer, J. Schou, R.I. Bush et al., Sol. Phys. 275, 207 (2012)

J. Schou, H.M. Antia, S. Basu et al., Astrophys. J. 505, 390 (1998)

M. Schulz, Astrophys. Space Sci. 24, 371 (1973)

R. Schwenn, Adv. Space Res. 26, 771 (2000)

R. Schwenn, B. Inhester, S.P. Plunkett et al., Sol. Phys. 175, 667 (1997) 
D.B. Seaton, D. Berghmans, B. Nicula et al., Sol. Phys. 286, 43 (2013)

M.A. Shay, J.F. Drake, M. Swisdak, W. Dorland, B.N. Rogers, Geophys. Res. Lett. 30, 1345 (2003)

N.R. Sheeley Jr., D.D.-H. Lee, K.P. Castro, Y.-M. Wang, N.B. Rich, Astrophys. J. 694, 1471 (2009)

N.R. Sheeley, Y.-M. Wang, S.H. Hawley et al., Astrophys. J. 484, 472 (1997)

R.N. Shelke, V.K. Verna, Bull. Astron. Soc. India 13, 53 (1985)

D.G. Sime, R.R. Fisher, R.C. Altrock, Astrophys. J. 336, 454 (1989)

E.J. Smith, J.R. Jokipii, J. Kóta, R.P. Lepping, A. Szabo, Astrophys. J. 533, 1984 (2000)

D. Spadaro, R. Ventura, G. Cimino, M. Romoli, Astron. Astrophys. 429, 353 (2005)

D. Spadaro, R. Susino, S. Dolei et al., Astron. Astrophys. 603, A35 (2017)

L. Strachan, R. Suleiman, A.V. Panasyuk, D.A. Biesecker, J.L. Kohl, Astrophys. J. 571, 1008 (2002)

L. Strachan, A.V. Panasiuk, J.L. Kohl, P. Lamy, Astrophys. J. 745, 51 (2012)

K.T. Strong, M. Bruner, T. Tarbell, A. Title, C.J. Wolfson, Space Sci. Rev. 70, 119 (1994)

K.T. Strong, J.L.R. Saba, B.M. Haisch, J.T. Schmelz (eds.), The Many Faces of the Sun - A summary of the Results from NASA's Solar Maximum Mission (Springer, Berlin, 1999)

S.T. Suess, A.-H. Wang, S.T. Wu et al., Space Sci. Rev. 87, 323 (1999)

X. Sun, J.T. Hoeksema, Y. Liu et al., Astrophys. J. 748, 77 (2012)

R. Susino, R. Ventura, D. Spadaro, A. Vourlidas, E. Landi, Astron. Astrophys. 488, 303 (2008)

L. Svalgaard, J. Geophys. Res. 77, 4027 (1972)

L. Svalgaard, J.M. Wilcox, Sol. Phys. 49, 177 (1975)

L. Svalgaard, J.M. Wilcox, Sol. Phys. 41, 461 (1976)

L. Svalgaard, I.G. Hannah, H.S. Hudson, Astrophys. J. 733, 49 (2011)

K. Tanaka, Astronomical Herald, vol. 74 (1981), p. 307

K. Tanaka, T. Watanabe, K. Nishi, K. Akita, Astrophys. J. Lett. 254, L59 (1982)

D. Telloni, E. Antonucci, M.A. Dodero, Astron. Astrophys. 472, 299 (2007a)

D. Telloni, E. Antonucci, M.A. Dodero, Astron. Astrophys. 476, 1341 (2007b)

D. Telloni, R. Bruno, V. Carbone, E. Antonucci, R. D’Amicis, Astrophys. J. 706, 238 (2009a)

D. Telloni, E. Antonucci, R. Bruno, R. D’Amicis, Astrophys. J. 693, 1022 (2009b)

D. Telloni, R. Ventura, P. Romano, D. Spadaro, E. Antonucci, Astrophys. J. 767, 138 (2013)

D. Telloni, E. Antonucci, S. Dolei et al., Astron. Astrophys. 565, A22 (2014)

D. Telloni, S. Giordano, E. Antonucci, Astrophys. J. Lett. 881, L36 (2019)

M.J. Thompson, J. Christensen-Dalsgaard, M.S. Miesch, J. Toomre, Annu. Rev. Astron. Astrophys. 41, 599 (2003)

A.F. Timothy, A.S. Krieger, G.S. Vaiana, Sol. Phys. 42, 135 (1975)

M. Tokumaru, D. Satonaka, K. Fujiki, K. Hayashi, K. Hakamada, Sol. Phys. 292, 41 (2017)

S. Tomczyk, S.W. McIntosh, S.L. Keil et al., Science 317, 1192 (2007)

Y. Uchida, A. McAllister, K.T. Strong, Y. Ogawara, T. Shimizu, R. Matsumoto, H.S. Hudson, Publ. Astron. Soc. Jpn. 44, L155 (1992)

I.G. Usoskin, S.V. Berdyugina, J. Poutanen, Astron. Astrophys. 441, 347 (2005)

M. Uzzo, Y.-K. Ko, J.C. Raymond et al., Astrophys. J. 585, 1062 (2003)

M. Uzzo, Y.-K. Ko, J.C. Raymond, Astrophys. J. 603, 760 (2004)

M. Uzzo, L. Strachan, A. Vourlidas, Astrophys. J. 671, 912 (2007)

G.S. Vaiana, J.M. Davis, R. Giacconi et al., Astrophys. J. Lett. 185, L47 (1973)

A.A. van Ballegooijen, M. Asgari-Targhi, A. Voss, Astrophys. J. 849, 46 (2017)

T. Van Doorsselaere, V.M. Nakariakov, E. Verwichte, Astrophys. J. Lett. 676, L73 (2008)

H.O. Vats, J.R. Cecatto, M. Mehta, H.S. Sawant, J.A.C.F. Neri, Astrophys. J. Lett. 548, L87 (2001)

J.-C. Vial, O. Engvold, Solar Prominences (Springer, Switzerland, 2015)

I. Virtanen, K. Mursula, Astron. Astrophys. 591, A78 (2016)

R. von Steiger, R. Wimmer Schweingruber, J. Geiss, G. Gloeckler, Adv. Space Res. 15, 3 (1995)

R. von Steiger, N.A. Schwadron, L.A. Fisk et al., J. Geophys. Res. 105, 27217 (2000)

R. von Steiger, T.H. Zurbuchen, D.J. McComas, Geophys. Res. Lett. 37, L22101 (2010)

W.J. Wagner, Astrophys. J. Lett. 198, L141 (1975)

R.W. Walsh, J. Ireland, Astron. Astrophys. Rev. 12, 1 (2003)

Y.-M. Wang, Space Sci. Rev. 172, 123 (2012)

Y.-M. Wang, Space Sci. Rev. 186, 387 (2014)

Y.-M. Wang, E. Robbrecht, Astrophys. J. 736, 136 (2011)

Y.-M. Wang, N.R. Sheeley Jr., Astrophys. J. 355, 726 (1990)

Y.M. Wang, N.R. Sheeley Jr., Astrophys. J. 430, 399 (1994)

Y.M. Wang, N.R. Sheeley Jr., J.H. Walters et al., Astrophys. J. Lett. 498, L165 (1998)

Y.-M. Wang, N.R. Sheeley, D.G. Socker et al., J. Geophys. Res. 105, 25133 (2000)

Y.-M. Wang, N.R. Sheeley Jr., N.B. Rich, Astrophys. J. 658, 1340 (2007)

Y.-M. Wang, E. Robbrecht, N.R. Sheeley Jr., Astrophys. J. 707, 1372 (2009) 
Y.-M. Wang, R. Grappin, E. Robbrecht, N.R. Sheeley Jr., Astrophys. J. 749, 182 (2012)

Y.-M. Wang, P.R. Young, K. Muglach, Astrophys. J. 780, 103 (2014)

H.P. Warren, N.A. Crump, I. Ugarte-Urra et al., Astrophys. J. 860, 46 (2018)

T. Wiegelmann, G.J.D. Petrie, P. Riley, Space Sci. Rev. 210, 249 (2017)

J.M. Wilcox, Comments Astrophys. Space Phys. 4, 141 (1972)

J.M. Wilcox, N.F. Ness, J. Geophys. Res. 70, 5793 (1965)

J.M. Wilcox, K.H. Schatten, A.S. Tanenbaum, R. Howard, Sol. Phys. 14, 255 (1970)

K. Wilhelm, W. Curdt, E. Marsh et al., Sol. Phys. 162, 189 (1995)

K. Wilhelm, E. Marsh, B.N. Dwivedi et al., Astrophys. J. 500, 1023 (1998)

G.L. Withbroe, Astrophys. J. 325, 442 (1988)

G.L. Withbroe, R.W. Noyes, Annu. Rev. Astron. Astrophys. 15, 363 (1977)

G.L. Withbroe, J.L. Kohl, H. Weiser, R.H. Munro, Space Sci. Rev. 33, 17 (1982)

J. Woch, W.I. Axford, U. Mall, B. Wilken, S. Livi, J. Geiss, G. Gloeckler, R.J. Forsyth, Geophys. Res. Lett. 24, 2885 (1997)

J.L. Xie, X.J. Shi, J. Zhang, Astrophys. J. 841, 42 (2017)

A.R. Yeates, D.H. Mackay, A.A. van Ballegooijen, Sol. Phys. 245, 87 (2007)

L. Zhang, K. Mursula, I. Usoskin, H. Wang, Astron. Astrophys. 529, A23 (2011)

L. Zhang, K. Mursula, I. Usoskin, Astron. Astrophys. 552, A84 (2013)

X.P. Zhao, J.T. Hoeksema, P.H. Scherrer, J. Geophys. Res. 110, A10 (2005)

L. Zhao, E. Landi, S.T. Lepri et al., Astrophys. J. 846, 135 (2017)

A.N. Zhukov, F. Saez, P. Lamy, A. Llebaria, G. Stenborg, Astrophys. J. 680, 1532 (2008) 